\title{
Attenuating Neurogenic Sympathetic Hyperreflexia Robustly Improves Antibacterial Immunity After Chronic Spinal Cord Injury
}

\author{
DEugene Mironets, ${ }^{1}{ }^{-}$Roman Fischer, ${ }^{2}$ Valerie Bracchi-Ricard, ${ }^{2}$ Tatiana M. Saltos, ${ }^{1}$ Thomas S. Truglio, ${ }^{1}$ \\ @Micaela L. O'Reilly, ${ }^{1}$ Kathryn A. Swanson, ${ }^{2}$ John R. Bethea, ${ }^{2}$ and $\odot$ Veronica J. Tom ${ }^{1}$ \\ ${ }^{1}$ Department of Neurobiology and Anatomy, Drexel University College of Medicine, Philadelphia, Pennsylvania 19129, and ${ }^{2}$ Department of Biology, \\ Drexel University, Philadelphia, Pennsylvania 19104
}

Spinal cord injury (SCI) disrupts critical physiological systems, including the cardiovascular and immune system. Plasticity of spinal circuits below the injury results in abnormal, heightened sympathetic responses, such as extreme, sudden hypertension that hallmarks life-threatening autonomic dysreflexia. Moreover, such sympathetic hyperreflexia detrimentally impacts other effector organs, including the spleen, resulting in spinal cord injury-induced immunodeficiency. Consequently, infection is a leading cause of mortality after SCI. Unfortunately, there are no current treatments that prophylactically limit sympathetic hyperreflexia to prevent subsequent effector organ dysfunction. The cytokine soluble tumor necrosis factor $\alpha(\operatorname{sTNF} \alpha)$ is upregulated in the CNS within minutes after SCI and remains elevated. Here, we report that commencing intrathecal administration of XPro1595, an inhibitor of sTNF $\alpha$, at a clinically feasible, postinjury time point (i.e., $3 \mathrm{~d}$ after complete SCI) sufficiently diminishes maladaptive plasticity within the spinal sympathetic reflex circuit. This results in less severe autonomic dysreflexia, a real-time gauge of sympathetic hyperreflexia, for months postinjury. Remarkably, delayed delivery of the sTNF $\alpha$ inhibitor prevents sympathetic hyperreflexia-associated splenic atrophy and loss of leukocytes to dramatically improve the endogenous ability of chronic SCI rats to fight off pneumonia, a common cause of hospitalization after injury. The improved immune function with XPro 1595 correlates with less noradrenergic fiber sprouting and normalized norepinephrine levels in the spleen, indicating that heightened, central sTNF $\alpha$ signaling drives peripheral, norepinephrine-mediated organ dysfunction, a novel mechanism of action. Thus, our preclinical study supports intrathecally targeting sTNF $\alpha$ as a viable strategy to broadly attenuate sympathetic dysregulation, thereby improving cardiovascular regulation and immunity long after SCI.

Key words: bacterial immunity; immunodeficiency; plasticity; soluble TNFa; spinal cord injury; sympathetic hyperreflexia

Significance Statement

Spinal cord injury (SCI) significantly disrupts immunity, thus increasing susceptibility to infection, a leading cause of morbidity in those living with SCI. Here, we report that commencing intrathecal administration of an inhibitor of the proinflammatory cytokine soluble tumor necrosis factor $\alpha$ days after an injury sufficiently diminishes autonomic dysreflexia, a real time gauge of sympathetic hyperreflexia, to prevent associated splenic atrophy. This dramatically improves the endogenous ability of chronically injured rats to fight off pneumonia, a common cause of hospitalization. This preclinical study could have a significant impact for broadly improving quality of life of SCI individuals.

\section{Introduction}

The incidence of infection and cardiovascular disease is higher in the spinal cord injury (SCI) population, particularly those with

Received Oct. 9, 2019; revised Nov. 11, 2019; accepted Nov. 14, 2019.

Author contributions: E.M., R.F., V.B.-R., T.M.S., T.S.T., and K.A.S. performed research; E.M., R.F., V.B.-R., T.M.S., M.L.O., and V.J.T. analyzed data; E.M. wrote the first draft of the paper;E.M., J.R.B., and V.J.T. wrote the paper; M.L.O. edited the paper; J.R.B. and V.J.T. designed research.

This work was supported by the National Institutes of Health (Grant R01 NS106908 to V.J.T. and J.R.B., Grant R01 NS111761 to J.R.B and V.J.T., Grant R01 NS085426 to V.J.T., and Grant R01 NS051709 to J.R.B.), the (raig H. Neilsen Foundation (Grant 382566 to V.J.T.), and the Drexel University Dean's Fellowship for Excellence in Collaborative or severe, high-level SCI, than in the able-bodied population (Garshick et al., 2005; Krassioukov and Claydon, 2006; Failli et al., 2012; Brommer et al., 2016). One major reason thought to
Themed Research Training (E.M.). We thank David E. Szymkowski of Xencor for supplying XPro1595; Jim Burns, Garth Ehrlich, Alison Carey, and their lab members for advice, training, and resources for the bacteriology experiments; Michael Lane and his lab members for advice and training for intubating the rodents; Alexander G. Rabchevsky for providing the MATLAB algorithm that served as the basis for what we used to analyze the naturally occurring events; Vladimir Zhukarev for assistance with the confocal microscope; the Marion Murray Drexel University Spinal Cord Research Center for use of its core facilities; and the Center for Neuroanatomy with Neurotrophic (supported by NIH Grant P40 0D010996) for providing the PRV-Bartha. 
underlie these potentially fatal issues is dysregulation of the sympathetic nervous system after SCI that results in pathological changes of multiple organ systems, including the spleen and vasculature (Alan et al., 2010; Zhang et al., 2013; Sauerbeck et al., 2015; Brommer et al., 2016; Prüss et al., 2017; Mironets et al., 2018). After SCI, plasticity within the spinal cord results in increased excitability of the spinal sympathetic reflex (SSR) circuit that produces excessive sympathetic output. We and others have shown that this sympathetic hyperreflexia, which is often triggered by below-level sensory stimuli (e.g., bladder and bowel distension), contributes to autonomic dysreflexia (AD), a lifethreatening cardiovascular disorder marked by episodes of extreme hypertension in response to sensory stimuli below the injury (Curt et al., 1997; West et al., 2015; Partida et al., 2016; Mironets et al., 2018), and splenic atrophy and dysimmunity, i.e., SCI-induced immunodeficiency syndrome (Zhang et al., 2013; Ueno et al., 2016; Prüss et al., 2017; Mironets et al., 2018). Unfortunately, there are no preventative treatments currently available.

The SSR circuit is comprised of neurons that relay sensory information to sympathetic preganglionic neurons (SPNs), the output neurons of the sympathetic nervous system that reside in thoracolumbar cord and project out of the spinal cord. In an intact CNS, SPN activity is coordinated by input from neurons within the brain and the spinal cord. If the level of SCI is high, the vast majority of the sympathetic nervous system is void of descending control and is regulated primarily by intraspinal input below the lesion. Additionally, injury induces maladaptive plasticity within the SSR circuit itself; increased arborization of mechanosensitive afferents and second order propriospinal interneurons may be responsible for recruitment of excitatory interneurons into the SSR circuit (Krenz and Weaver, 1998; Krenz et al., 1999; Weaver et al., 2001, 2002; Krassioukov et al., 2002; Hou et al., 2008; Ueno et al., 2016). While there is increasing understanding of what changes occur in the SSR circuit after SCI (Cameron et al., 2006; Rabchevsky et al., 2011), the mechanisms by which injury induces these changes remains unclear.

Cytokine signaling has been postulated to contribute to excitability of circuits. In addition to orchestrating immune function, cytokines play important roles in modulating synaptic function during development (Wheeler et al., 2014) and disease (Cerri et al., 2017). Is it possible that upregulated cytokine signaling, which is seen after SCI (Lee et al., 2000; Donnelly and Popovich, 2008), mediates plasticity of the SSR circuit to result in sympathetic hyperreflexia? Indeed, we demonstrated that persistent, proinflammatory soluble (s) TNF $\alpha /$ TNFR1 signaling below a highlevel complete SCI is a critical mediator of SSR circuit plasticity (Mironets et al., 2018). However, sTNF $\alpha$ is upregulated within minutes after SCI (Wang et al., 1996; Bethea et al., 1999), so it can impact SSR circuit almost immediately. Here, we assessed whether commencing administration of XPro1595, a biologic that inhibits sTNF $\alpha$ binding to TNFR1 (Steed et al., 2003) at a realistic, clinically feasible time frame, i.e., $3 \mathrm{~d}$ after SCI, prophylactically attenuates SCI-induced sympathetic hyperreflexia and ensuing immunodeficiency to improve the endogenous ability of chronic SCI rodents to fend off bacterial pneumonia.

The authors declare no competing financial interests.

Please address all correspondence to John R. Bethea at jrb445@drexel.edu or Veronica J. Tom at vit25@drexel.edu.

https://doi.org/10.1523/JNEUROSCI.2417-19.2019

Copyright $\odot 2020$ the authors

\section{Materials and Methods}

Animal use. Adult, female Wistar rats ( 225-250 g; Charles River Laboratories) were used for all experiments. Animals were given unlimited access to food and water and used in accordance with Drexel University Institutional Animal Care and Use Committee and National Institutes of Health guidelines for experimentation with laboratory animals. Animals acclimated for a least 1 week after arrival before any procedure was done and before being randomly assigned to treatment groups. All surgical procedures were performed under aseptic conditions using sterilized instruments. During surgery, animals were given $3 \mathrm{ml}$ Lactated Ringer's, ampicillin $(200 \mathrm{mg} / \mathrm{kg}$ ), and slow-release buprenorphine (ZooPharm; $0.05 \mathrm{mg} / \mathrm{kg}$ ) or meloxicam (Putney; $1 \mathrm{mg} / \mathrm{kg}$ ) for analgesia. After surgery, animals were placed in a postoperative cage on a heating pad and monitored until they became alert and responsive. All animals with spinal transections had their bladders manually expressed at least twice a day for the duration of the study.

Radiotelemeter implantation to monitor hemodynamics. As we did previously (Hou et al., 2013; Mironets et al., 2018), radiotelemeter pressure transducers (model HD-S11; Data Sciences International) were surgically transplanted into the descending aorta via the femoral artery to detect cardiovascular parameters of naive animals at least 1 week before spinal transection (Tx) to give the animals ample recovery time after the implantation surgery (Rabchevsky et al., 2012). Blood pressure was monitored to ensure patency of the probes and to obtain preinjury baseline data.

Thoracic spinal transection and osmotic minipump implantation. One week after telemeter implantation, animals were deeply anesthetized using isoflurane, and the spinal cord at thoracic level 3 (T3) was exposed by dorsal laminectomy of the T2 vertebral processes [overlying T3 spinal cord level; (Wu et al., 2015; Xu et al., 2015)]. After a small incision was made in the dura overlying $\mathrm{T} 3 \mathrm{using}$ a microknife, a $\sim 2 \mathrm{~mm}$ long segment of the spinal cord was removed by vacuum aspiration using a pulled glass micropipette. Lesion completeness was verified visually.

Three days after Tx, a small incision was made in the dura above T8 spinal cord. As we did before (Mironets et al., 2018), an intrathecal catheter (ReCathCo) attached to an osmotic minipump (Alzet, no. 2002) containing saline or XPro 1595 (10 mg/ml; provided by Inmune Bio) was carefully threaded subdurally until the end lay just caudal to the Tx site to continuously deliver saline or $60 \mu \mathrm{g}$ per day of XPro1595. This daily dose successfully inhibits intraspinal plasticity below a complete SCI (Mironets et al., 2018). Because the pumps have 28 -day delivery durations, the osmotic minipumps were replaced 4 weeks after implantation.

Blood pressure recordings to assess naturally occurring autonomic dysreflexia. Rodents ( $n=18-24$ /group) were individually placed in cages and positioned on telemetry receivers (RC-1; Data Sciences International). Baseline recordings of all animals after telemeter implantation but before SCI were obtained to ensure that HR and MAP values were within a normal range, confirming that the catheters were not occluded. At every other week from 2 to 8 weeks post-SCI, MAP and HR were monitored continuously while animals moved freely in their cages for $24 \mathrm{~h}$ (MAP and HR values sampled every $2 \mathrm{~s}$; Dataquest A.R.T. acquisition software, Data Sciences International). As we did previously (Mironets et al., 2018), to identify naturally occurring $\mathrm{AD}$ events, these data from individual animals at each time point were analyzed in MATLAB. Rolling MAP and HR baselines were established by continuously averaging a 6 min period. $\mathrm{AD}$ events were defined as when MAP was at least $20 \mathrm{mmHg}$ greater than baseline for at least $30 \mathrm{~s}$ and was accompanied by bradycardia of at least 20 beats per minute (bpm). All "detected" events were visually verified by a blinded observer according to the criterion described above. All false-positive events were disregarded. Any events that occurred within 2 min of each other were considered to be 1 event. Any detected events within 15 min of manual bladder expression were not included in additional comparative analyses. Once an event was verified, the average MAP during the event, the change in MAP from baseline, the HR during the event, and the duration of the bout was calculated.

Colorectal distension to induce autonomic dysreflexia. At 2, 4, 6 and 8 weeks post-transection, BP and HR were monitored before, during, and after colorectal distension (CRD) in unanesthetized rats, a well estab- 
lished technique that reliably induces an $\mathrm{AD}$ episode (Mayorov et al., 2001; Cameron et al., 2006). As we did previously (Hou et al., 2013; Mironets et al., 2018), a silicone balloon-tipped catheter (2-way pediatric Foley cathether, 10 French, $3 \mathrm{cc}$, Coloplast) was inserted $2 \mathrm{~cm}$ inside the rectums of all T3Tx rats treated with saline or XPro1595 and secured to the tails with tape. Animals were acclimated to the catheter for at least 30 min. AD was induced by inflating a balloon catheter with $2.0 \mathrm{ml}$ of air for $1 \mathrm{~min}$. Two or three trials were conducted per animal per time point, with an intertrial interval of at least $20 \mathrm{~min}$. The difference between the baseline MAP and the CRD-induced MAP and the time it took for BP to return to baseline values was calculated for each trial and averaged per animal per time point.

Streptococcus pneumoniae infection post-SCI. S. pneumoniae is a strain of bacteria that successfully infects Wistar rats (Feldman et al., 1991; Brandt et al., 2008). Serotype 3 S. pneumoniae [(Klein) Chester; ATCC 6303] was grown in BHI medium to an optical density $\left(\mathrm{OD}_{470}\right)$ of 0.4 to 0.6 and diluted to the appropriate concentration in sterile PBS. The bacteria suspension was plated on blood agar plates to confirm quantity and viability of the bacteria. The exact concentration of the bacteria suspension was used to normalize the bacterial loads in the lungs. For infection, uninjured rats or T3Tx-Saline or -XPro1595 rats 8 weeks postinjury ( $n=5-7 /$ group) were anesthetized with isoflurane and then suspended at a $60^{\circ}$ angle by the two front upper teeth by a wire attached to a Plexiglas support. A $16 \mathrm{G}$ peripheral venous catheter (Braun) was used as a tubus and a $0.5 \mathrm{~mm}$ optical fiber (Biotex, Inc) attached to a cold light source was used for illumination (MacDonald et al., 2009). The tongue was pulled out with a small spatula and the rat was carefully intubated. S. pneumoniae [5000 colony-forming units (CFUs) in $100 \mu \mathrm{l}$ ] was then administered into the lung. The size of the inoculum was confirmed by quantitative cultures. After intubation, animals were placed in heated cages for $10 \mathrm{~d}$. Body temperature, weight, and respiratory rate were recorded daily (at $24 \mathrm{~h}$ intervals postinfection) to track sickness behavior. Animals that lost $>15 \%$ body weight were killed.

Ten days after infection, animals were killed. Lungs were extracted and homogenized in $1 \mathrm{ml}$ of sterile PBS and plated on blood agar plates (Hardy Diagnostics) in serial dilutions of 1:10, 1:100, and 1:1000. CFUs were counted after $48 \mathrm{~h}$ incubation and the total number of CFUs per lung was calculated. To quantify CFUs, images of blood agar plates were thresholded. The image was then converted to a binary image to count individual particles (ImageJ).

FluoroGold (FG) and pseudorabies virus injections. One week before being killed, T3Tx rodents ( $n=4-5 /$ group) were injected $0.4 \mathrm{ml}$ intraperitoneally of $0.5 \%$ FG (diluted in $0.9 \%$ sterile saline) to label SPNs (Derbenev et al., 2010). To label sympathetically associated neurons, attenuated pseudorabies virus-Bartha encoding for a GFP-reporter (PRV-152; NIH-supported Center for Neuroanatomy with Neurotrophic Viruses) was injected bilaterally into the kidneys $4 \mathrm{~d}$ before being killed, similar to what was done in (Duale et al., 2009). Bilateral incisions were made and kidneys were exposed. With the aid of a dissecting stereomicroscope, $3 \mu \mathrm{l}$ of PRV-152 $\left(10^{8}\right.$ plaque-forming units $\left./ \mathrm{ml}\right)$ was slowly injected into 3 sites along the longitudinal midline of the convex surface of the kidney of each kidney of T3Tx rats using a 30G Hamilton syringe with a pulled glass micropipette with a beveled tip. Animals were killed and perfused $96 \mathrm{~h}$ later.

Intermittent colorectal distension to activate the SSR circuit and induce cFos expression. To activate the SSR circuit (as indicated by histological assessment of cFos induction; see histology description below), some T3Tx-Saline or T3Tx-XPro1595 animals ( $n=4$ /group) were given intermittent CRD over an extended period of time at 8 weeks postinjury (Landrum et al., 2002; Hou et al., 2008; Ueno et al., 2016). Briefly, a balloon catheter inserted into the rectum was inflated with $2.0 \mathrm{ml}$ of air for $30 \mathrm{~s}$ and then deflated for $60 \mathrm{~s}$. This $90 \mathrm{~s}$ cycle was repeated 30 times for a total of $45 \mathrm{~min} .1 .5 \mathrm{~h}$ after the last round of CRD, animals were killed and perfused with ice-cold $0.9 \%$ saline followed by $4 \%$ paraformaldehyde in PBS.

Flow cytometry. Flow cytometry analysis was performed as we did previously (Mironets et al., 2018). Rats ( $n=5-7 /$ group) were killed 8 weeks after SCI, spleens were extracted, dissociated through a $40 \mu \mathrm{m}$ cell strainer and collected in $10 \mathrm{ml}$ MACS buffer (PBS, 0.5\% BSA, $2 \mathrm{~mm}$
EDTA). Splenocytes were harvested by centrifugation $(300 \times g, 5 \mathrm{~min})$, washed once with $10 \mathrm{ml}$ of MACS buffer and incubated in $3 \mathrm{ml}$ of ACK Lysing Buffer (Thermo Fisher Scientific) for 5 min to deplete red blood cells. After washing with $10 \mathrm{ml}$ of MACS buffer, splenocytes were then stained for different immune cell markers using fluorescence-labeled antibodies (Miltenyi Biotec). For staining of cytoplasmic proteins, the FoxP3 Staining Buffer Set was used according to manufacturer's instructions (Miltenyi Biotec; \#130-093-142). Data were acquired using a FACS Canto (BD Biosciences) and analyzed with FlowJo (TreeStar).

ELISA. Naive, T3Tx-Saline, or T3Tx-XPro1595 rats at 8 weeks postSCI ( $n=3-7 /$ group) were killed and fresh lumbar spinal cord or spleen tissue was immediately extracted and flash-frozen in liquid nitrogen and stored at $-80^{\circ} \mathrm{C}$ until further processing. As previously described (Bethea et al., 1999; Bracchi-Ricard et al., 2013), samples were homogenized in RIPA buffer ( $0.01 \mathrm{M}$ sodium phosphate $\mathrm{pH} 7.2,0.15 \mathrm{M} \mathrm{NaCl}, 1 \%$ NP40, $1 \%$ sodium deoxycholate, $0.1 \%$ SDS, 2 mm EDTA) supplemented with Roche complete protease inhibitor mixture, mixed end-over-end at $4^{\circ} \mathrm{C}$ for $30 \mathrm{~min}$, and centrifuged at $14,000 \mathrm{rpm}$ for $10 \mathrm{~min}$ at $4^{\circ} \mathrm{C}$. The supernatants were transferred to fresh tubes and stored at $-80^{\circ} \mathrm{C}$. Protein quantification of each sample was performed using DC Protein Assay (Bio-Rad). TNF $\alpha$ (Abcam), corticosterone (ENZO), and NE (MyBioSource) ELISA kits and were used to measure expression levels of sTNF $\alpha$, corticosterone and NE per the manufacturer's instructions. Samples were measured at an absorbance of (450) nm cuvettes were washed five times with washing buffer [PBS, pH 7.4, containing $0.1 \%$ (v/v) Tween 20] after each step. As a reference for quantification, a standard curve was established by a serial dilution.

Histology. Naive animals and T3Tx-Saline and -XPro1595 animals 8 weeks post-SCI ( $n=4-5$ /group) were killed and perfused with $0.9 \%$ saline followed by $4 \%$ paraformaldehyde. The spinal cords and spleens were dissected out, postfixed overnight at $4^{\circ} \mathrm{C}$, and cryoprotected in sucrose before cutting $30 \mu \mathrm{m}$ transverse sections of lumbar spinal cord and spleen or $30 \mu \mathrm{m}$ longitudinal sections of T8-T13 cord on a cryostat. Sections were blocked in 5\% normal goat serum, 10\% BSA, 0.1\% Triton $\mathrm{X}-100$ in PBS for $1 \mathrm{~h}$.

After blocking, sections were incubated with appropriate primary antibodies overnight at $4^{\circ} \mathrm{C}$. Longitudinal lower thoracic sections (T8T13) were incubated anti-c-Fos (Santa Cruz Biotechnology) to visualize neurons activated by CRD and anti-GFP (Millipore) to visualize neurons trans-synaptically traced via PRV-152. SPNs were labeled via intraperitoneal FG injections and were visible microscopically under UV excitation without any staining. Transverse lower lumbar sections (L6) were incubated with anti-GFP, anti-c-Fos, anti-CGRP (Peninsula Laboratories) to visualize nociceptive primary afferents axons. Transverse spleen sections were incubated with anti-CD45 (Novus) and anti-tyrosine hydroxylase (TH; Millipore) to visualize splenocytes and noradrenergic fibers, respectively. Sections were washed in PBS, incubated with appropriate AlexaFluor-conjugated secondary antibodies (Invitrogen) for $2 \mathrm{~h}$ at room temperature, washed in PBS, mounted onto slides, and coverslipped with FluorSave (EMD Chemical). Sections were stained at the same time. For each of the antibodies listed above, images of 4 equivalent sections per animal were captured on Olympus BX51 and Leica DM5500B epifluorescent microscopes and a Leica TCS SP2 confocal microscope equipped with a Leica DMRE microscope using the same exposure time per antibody.

All image analyses were conducted using ImageJ. In thoracic sections containing $\mathrm{FG}^{+}, \mathrm{GFP}^{+}$, and $\mathrm{cFos}^{+}$near the IML and central canal or lumbar sections containing $\mathrm{GFP}^{+} / \mathrm{cFos}^{+}$near the dorsal horn and/or around the central canal, an intensity threshold was applied to each image. The same threshold values were used for each antibody. We assessed the extent of immunoreactivity for cFos by counting the number of $\mathrm{cFos}^{+}$nuclei in identically sized regions of dorsal horn and around the central canal (in lumbar sections) or in intermediate gray adjacent to the IML. To assess the extent of GFP and FG immunoreactivity, all GFP ${ }^{+}$ and $\mathrm{FG}^{+}$cells within a circumscribed area in thoracic cord through the section plane were counted.

To quantify CGRP immunoreactivity, thresholded pixel areas were determined in equally sized regions encompassing laminae I-IV or lamina VII/X (i.e., dorsal gray commissure around the central canal). 
Table 1. Summary of animal numbers for each experimental outcome measure

\begin{tabular}{lc}
\hline Experimental outcome measure & $\begin{array}{l}\text { No. of animals } \\
\text { per group }\end{array}$ \\
\hline ELISA & $3-7$ \\
CGRP + histology & $3-4$ \\
Assessment of recruitment and activation of neurons in the SSR circuit & 4 \\
Assessment of autonomic dysreflexia & $18-24$ \\
Spleen and splenocyte analysis & $5-6$ \\
Spleen histology & $4-5$ \\
S.pneumoniae infection challenge & $5-7$ \\
\hline
\end{tabular}

To quantify white pulp area, white pulp regions were outlined and assessed for area (in square micrometers). To quantify $\mathrm{TH}^{+}$axonal arbors in splenic white pulp regions, thresholded pixel areas were determined in selected white pulp regions. $\mathrm{TH}^{+}$splenocytes were excluded.

Experimental design and statistical analyses. Animals were randomly assigned to treatment groups. Sample sizes were determined based upon our previous published studies using similar techniques (summarized in Table 1). All analyses were performed blinded to treatment group. To determine differences between two groups, a Student's $t$ test was performed. A two-way ANOVA and post hoc Fisher's LSD tests were performed to ascertain differences between groups over time, i.e., hemodynamic recording analyses. A one-way ANOVA and post hoc Fisher's LSD tests were performed to assess for differences between three or more groups in which time was not a consideration, i.e., histological and biochemical analyses. A $p$-value $<0.05$ was considered significant. All statistical tests were performed using GraphPad Prism 7.

\section{Results}

\section{TNF $\boldsymbol{\alpha}$ is chronically upregulated below a T3Tx}

We assessed whether TNF $\alpha$ levels was persistently upregulated in lumbar cord, where we and others have observed enhanced plasticity of SSR circuits associated with AD and immunosuppression after a T3Tx (Krenz et al., 1999; Cameron et al., 2006; Hou et al., 2009; Kroner et al., 2014; Mironets et al., 2018). Using ELISA, we found that there was some TNF $\alpha$ in naive lumbar tissue (533.1 \pm $104.8 \mathrm{pg} / \mathrm{mg}$ tissue) but that expression was significantly higher in lumbar tissue 8 weeks after T3Tx $(1682.0 \pm 108.7 \mathrm{pg} / \mathrm{mg}$ tissue; $\left.t_{(8)}=6.284, p=0.0002\right)$.

\section{Delayed sTNF $\alpha$ inhibition decreases plasticity of CGRP ${ }^{+}$ primary afferents after chronic SCI}

After SCI, the SSR circuit becomes more sensitive to and is activated by noxious sensory stimuli below the injury. One of the maladaptive changes thought to underlie this is increased density of calcitonin-gene related peptide $(\mathrm{CGRP})^{+}$, nociceptive primary afferents in deeper layers of spinal cord (Marsh et al., 2002; Cameron et al., 2006) where they synapse on interneurons that then relay nociceptive information to the SPNs in the IML (Joshi et al., 1995; Hou et al., 2008). Indeed, the degree of CGRP ${ }^{+}$ expression positively correlates with the magnitude of $\mathrm{AD}$ episodes; inhibiting injury-induced plasticity of $\mathrm{CGRP}^{+}$fibers attenuates AD (Krenz et al., 1999; Weaver et al., 2001; Marsh et al., 2002; Cameron et al., 2006). We sought to determine whether inhibiting sTNF $\alpha$ diminishes plasticity of these sensory fibers known to activate the SSR circuit. To do this, we initiated intrathecal administration of the sTNF $\alpha$ dominant-negative biologic XPro1595 at a clinically feasible, post-SCI time point (i.e., $3 \mathrm{~d}$ after complete T3Tx).

We examined CGRP ${ }^{+}$immunoreactivity in lower lumbar spinal cord from naive or T3Tx-Saline and T3Tx-XPro1595 animals 8 weeks post-SCI. We focused on this region because primary afferents carrying sensory information from the colon, whose activation is a common prompt for sympathetic events in the human SCI population and in rodent SCI models [e.g., with colorectal distension (CRD)], enter the spinal cord at these levels (Landrum et al., 2002). CGRP ${ }^{+}$afferents normally project to superficial Rexed Laminae (I-II) of dorsal horn and, to a lesser degree, to deeper dorsal horn laminae (Fig. 1a) and Laminae $\mathrm{VII} / \mathrm{X}$ around the central canal (Fig. 1e). All groups (naive, T3TxSaline, T3Tx-XPro1595) had a similar extent of CGRP ${ }^{+}$immunoreactivity in the dorsal horn (Fig. $1 a-d ; p=0.5528$ ). However, T3Tx-Saline animals had more CGRP immunoreactivity around the central canal compared with both naive and T3Tx-XPro1595 animals (Fig. $1 e-h ; F_{(2,23)}=4.376, p=0.0245$, post hoc vs naive $p=0.0322$, vs T3Tx-XPro1595 $p=0.0128)$. Furthermore, T3TxXPro1595 animals had similar levels to naive animals (Fig. 1e,g, $h$; $p=0.8200)$. These data indicate that injury-induced sTNF $\alpha$ signaling may increase sensitivity of the SSR circuit by triggering $\mathrm{CGRP}^{+}$plasticity onto interneurons around the central canal and that delivering XPro1595 $3 \mathrm{~d}$ after SCI is sufficient to prevent this increase in $\mathrm{CGRP}^{+}$labeling density.

\section{Delayed sTNF $\alpha$ inhibition reduces the recruitment and activation of interneurons in the spinal sympathetic reflex circuit}

After SCI, the SSR circuit becomes more sensitive to sensory stimuli below the injury. One change that has been implicated in hyperexcitability of the SSR circuit is the recruitment and activation of interneurons into the circuit (Krassioukov et al., 2002; Landrum et al., 2002; Hou et al., 2008; Ueno et al., 2016). We sought to determine whether inhibiting sTNF $\alpha$ starting $3 \mathrm{~d}$ after SCI decreases injury-induced, increased activation of interneurons in the SSR circuit. We injected PRV-152 into the kidneys of T3Tx-Saline and T3Tx-XPro1595 animals at 8 weeks post-SCI to transsynaptically trace sympathetically associated neurons. We also injected FG intraperitoneally, an established means to label SPNs (Hou et al., 2008). To further identify neurons that are within the SSR circuit, the same animals were subjected to CRD $4 \mathrm{~d}$ after PRV injection, just before being killed. CRD is an established means to activate the SSR circuit and induce cFos, a marker of neuronal activation (Landrum et al., 2002; Hou et al., 2008), in sympathetically correlated neurons. Thus, we were able to identify SPNs $\left(\mathrm{FG}^{+}\right)$or interneurons $\left(\mathrm{FG}^{-}\right)$that are associated with sympathetic function $\left(\mathrm{PRV}^{+}\right)$that were activated by the visceral sensory stimulus $\left(\mathrm{cFos}^{+}\right)$.

Lumbar interneurons in laminae VII/X that receive afferent input relay that sensory information rostrally toward the SPNs in the IML (Clarke et al., 1998; Matsushita, 1998; Tang et al., 2004; Hofstetter et al., 2005; Cameron et al., 2006). We found significantly more $\mathrm{PRV}^{+}$neurons in lumbar spinal cord in T3Tx-Saline animals than in T3Tx-XPro1595 ones (Fig. $2 a, d, g ; t_{(22)}=2.989$, $p=0.0068)$, indicating that XPro1595 diminishes the recruitment of interneurons into the SSR circuit at this level. There were also more interneurons that responded to CRD in the salinetreated animals (Fig. $2 b, e, h ; t_{(22)}=4.546, p=0.0002$ ). Moreover, in XPro1595-treated animals, there were fewer lumbar sympathetically associated interneurons (i.e., $\mathrm{PRV}^{+} / \mathrm{cFos}^{+}$) that were activated by CRD (Fig. $2 c, f, i ; t_{(22)}=2.197, p=0.0389$ ).

In thoracic spinal cord, there were more sympathetically associated interneurons in close proximity to SPNs within the IML that were activated by CRD (i.e., $\mathrm{PRV}^{+} / \mathrm{CFOs}^{+} / \mathrm{FG}^{-}$) in T3TxSaline animals than in T3Tx-XPro1595 ones (Fig. $2 j-r ; t_{(27)}=$ $2.096, p=0.0456)$. Although the number of activated SPNs was similar between groups (Fig. $2 s ; p=0.4891$ ), the total number of activated interneurons in the SSR circuit as well as the ratio acti- 

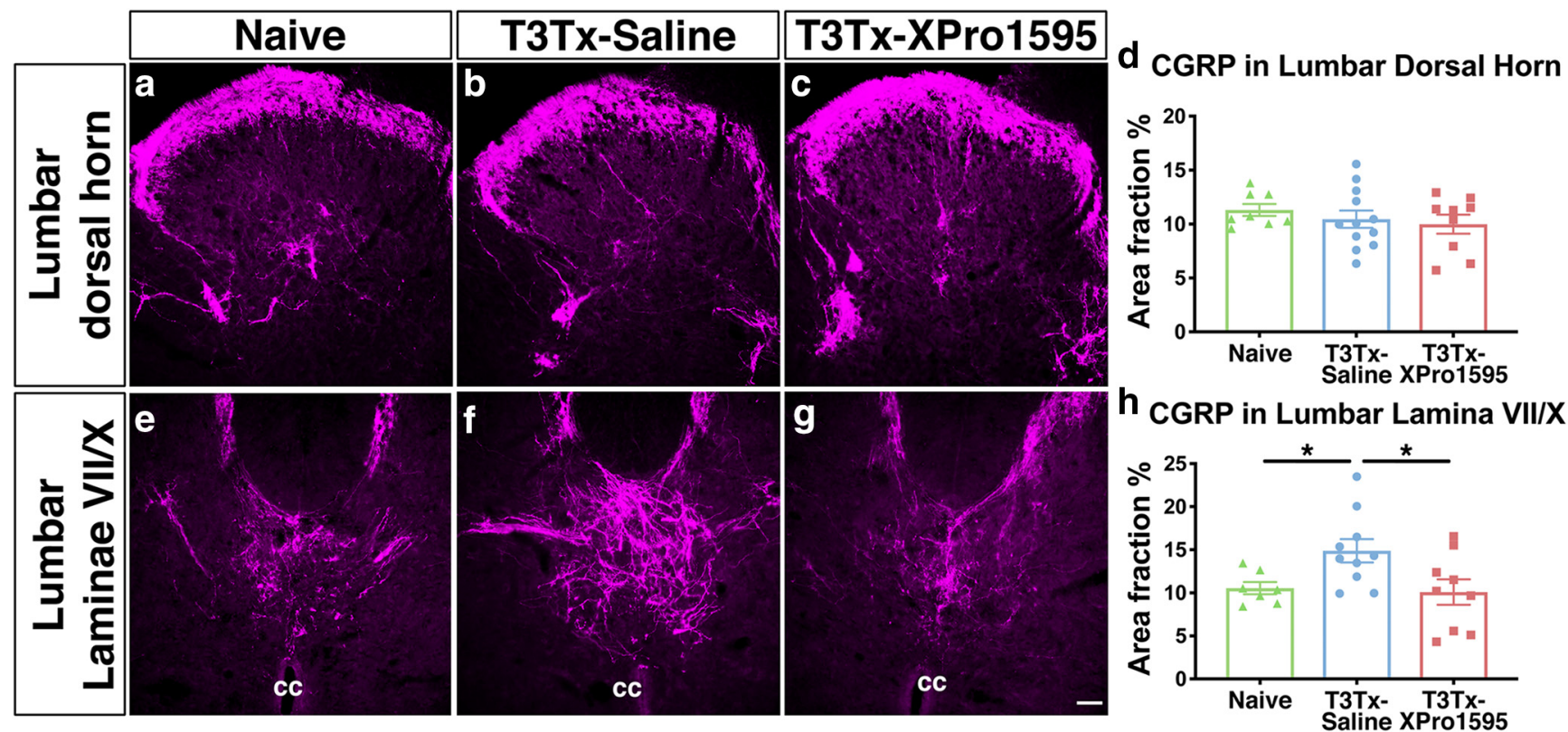

Figure 1. Delayed inhibition of STNF $\alpha$ after T3Tx diminishes CGRP ${ }^{+}$afferent plasticity near the central canal in lower lumbar cord. Representative images of transverse sections of $L 6$ lumbar cord from T3TX-Saline animals and T3TX-XPro1595 animals 8 weeks post-injury and naive, uninjured animals immunostained for CGRP. $\boldsymbol{a}$-d, T3Tx-Saline (b) and T3Tx-XPro1595 animals (c) had similar levels of CGRP ${ }^{+}$expression in dorsal horn as naive, uninjured animals $(\boldsymbol{a}, \boldsymbol{d}) \cdot \boldsymbol{e}-\boldsymbol{h}$, There was increased CGRP ${ }^{+}$immunoreactivity in T3TX-Saline animals $(\boldsymbol{f})$ in Laminae VII/X around the central canal compared with both naive animals $(\boldsymbol{e})$ and T3TX-XPro1595 animals $(\boldsymbol{g}, \boldsymbol{h})$. T3TX-XPro1595 animals had normal levels of CGRP expression near the central canal. $n=3 / 4$ per group. Mean \pm SEM ${ }^{*} p<$ 0.05. cc: central canal. Scale bar, $50 \mu \mathrm{m}$.

vated interneurons to SPNs was higher in the T3Tx-Saline animals (Fig. $2 r, t ; t_{(27)}=2.067, p=0.0485$ ).

Altogether, these data indicate that inhibiting $\operatorname{sTNF} \alpha$ with XPro1595 decreases the recruitment and excitability of lumbar and thoracic interneurons in the SSR circuit after SCI.

Initiating intrathecal administration of XPro1595 $3 \mathrm{~d}$ after SCI diminishes the severity of sympathetic hyperreflexia While the data above suggest blocking sTNF $\alpha$ after T3Tx attenuates anatomical plasticity of the SSR circuit, does this have functional implications? To determine this, we took advantage of the fact that activation of the SSR circuit in T3Tx animals manifests almost immediately as an AD episode. Thus, we can use hemodynamic measures to not only assess sympathetically mediated $\mathrm{AD}$ but to also serve as a virtually instantaneous readout of SSR circuit activity.

We implanted a radiotelemetry pressure transducer into the descending aortas of adult rats before T3Tx and intrathecal treatment with saline or XPro1595, allowing for longitudinal monitoring of mean arterial pressure (MAP) and heart rate (HR) in the same animals at multiple time points. Hemodynamics were assessed before injury and at biweekly time points up to 8 weeks after SCI. SCI resulted in a decrease in baseline MAP at 8 weeks for both T3Tx-Saline and T3Tx-XPro1595 animals (Fig. 3a; $F_{(4,116)}=12.91, p<0.0001$; post hoc, vs 8 weeks T3Tx-Saline $p<$ 0.0001 ; vs 8 weeks T3Tx-XPro1595 $p=0.0271$ ), similar to what we saw previously (Mironets et al., 2018). There were no differences in MAP between experimental groups at any time point. SCI also resulted in increased HR at 8 weeks for both groups compared with preinjury baseline (Fig. $3 b ; F_{(4,116)}=6.355, p=$ 0.0001 ; post hoc, vs 8 weeks T3Tx-Saline $p=0.0145$; vs 8 weeks T3Tx-XPro1595 $p=0.0003$ ).

We also examined hemodynamic parameters before, during and after subjecting the T3Tx-Saline and -XPro1595 animals to CRD, a well established experimental technique used to activate the SSR circuit and trigger AD virtually instantaneously (Krassioukov and Weaver, 1995; Krenz et al., 1999; Cameron et al., 2006; Hou et al., 2013). CRD quickly elicited AD events in both T3Tx-Saline and T3Tx-XPro1595 animals (Fig. 3c-d). In T3TxSaline animals, there was a significant increase in the magnitude of CRD-induced hypertension between 2 and 8 weeks (Fig. 3e; $F_{(3,102)}=5.976, p=0.0009$; post hoc, 2 weeks T3Tx-Saline vs 8 weeks T3Tx-Saline $p=0.0099)$. The magnitude of CRD-induced MAP spikes in T3Tx-XPro1595 animals also progressively increased over the course of weeks 2 through 8 (Fig. $3 e ; F_{(3,102)}=$ 5.976, $p=0.0009$; post hoc, 2 weeks T3-XPro1595 vs 8 weeks T3Tx-XPro1595 $p=0.0040$ ). However, the MAP spikes in the XPro1595-treated animals were significantly smaller compared with those in the T3Tx-Saline rats at all post-SCI time points (Fig. $3 e ; F_{(1,102)}=35.89, p<0.0001$; post hoc, at 2 weeks $p=0.0025$, at 4 weeks $p<0.0001$, at 6 weeks $p=0.0145$, at 8 weeks $p=0.0221$ ). XPro 1595 treatment also markedly reduced the time to return to baseline MAP after the CRD was relieved at all time points (Fig. $3 f ; F_{(1,118)}=40.57, p<0.0001$, post hoc, at 2 weeks $p=0.0052$, at 4 weeks $p=0.0004$, at 6 weeks $p=0.0195$ at 8 weeks $p=0.0001$ ). We also found that T3Tx-XPro1595 animals had significant less reflexive bradycardia during the hypertensive $\mathrm{AD}$ than $\mathrm{T} 3 \mathrm{Tx}-$ Saline animals at 4 - and 6-weeks after injury (Fig. $3 g ; F_{(1,102)}=$ $13.83, p=0.0003$, post hoc at 4 weeks $p=0.0303$, at 6 weeks $p=$ $0.0006)$.

People (and rodents) with high-level injuries often experience numerous $\mathrm{AD}$ events per day due to common complications associated with SCI [e.g., pressure sores, full bladders, constipation; (Rabchevsky et al., 2012; Zhang et al., 2013; West et al., 2015)]. To further assess the degree to which XPro1595 diminishes AD, we determined the number of daily "naturally occurring", nonexperimentally induced AD bouts. At each biweekly time point, we recorded animals' MAP and HR over a continuous $24 \mathrm{~h}$ period while they moved freely in their cages. AD events were detected using an established algorithm (Mironets et al., 2018) that sifts 

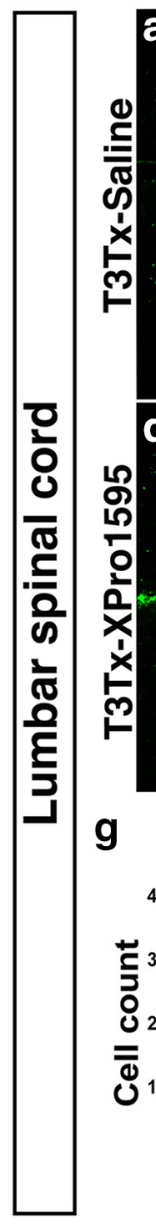

a

PRV
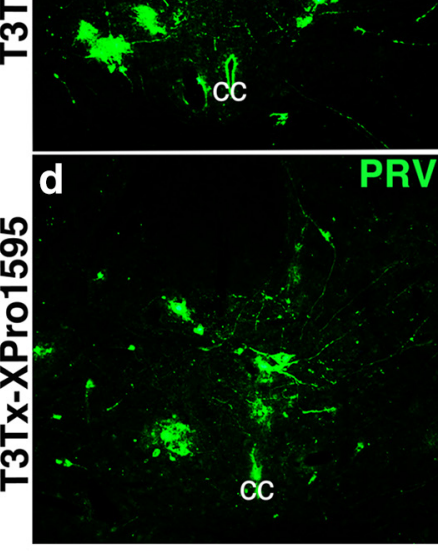

g

\begin{abstract}
PRV+ cells
\end{abstract}

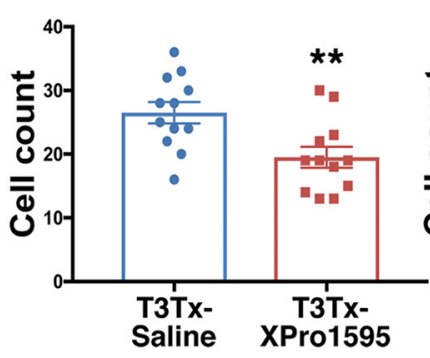

h

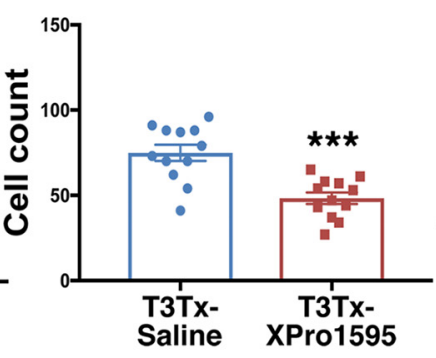

cC
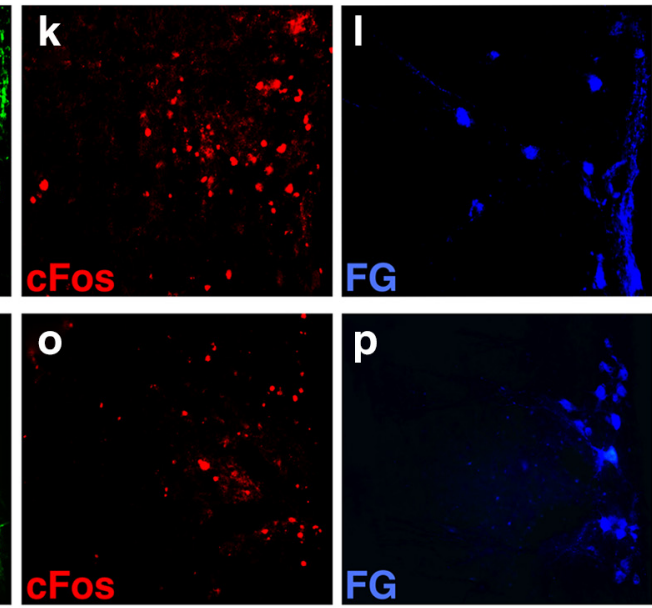

$\mathbf{S}$

$\mathrm{PRV}^{+} / \mathrm{CFOS}^{+} / \mathrm{FG}^{+}$SPNs

r $\quad \mathrm{PRV}^{+} / \mathrm{cFos}^{+} / \mathrm{FG}^{-}$interneurons
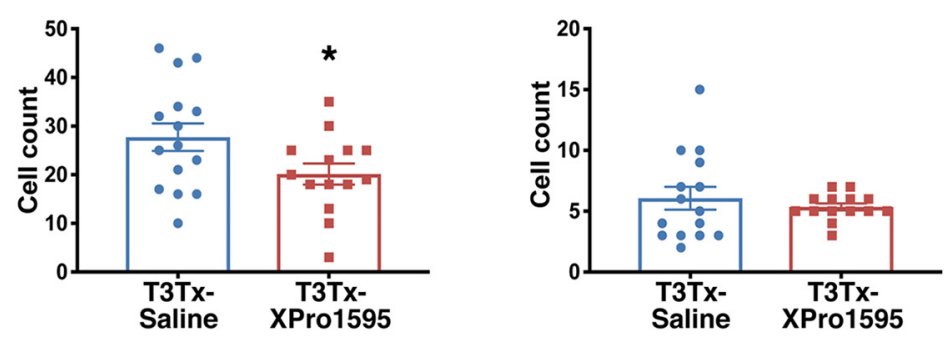

Figure 2. Delayed inhibition of spinal sTNF $\alpha$ decreases the recruitment and colorectal distension-elicited activation of interneurons in the SSR circuit. PRV (green) was used to identify neurons within the SSR circuit and FG (blue) was used to label SPNs. Repeated, intermittent CRD was given to T3TX-Saline or -XPro 1595 animals 8 weeks post-SCI to activate neurons within the SSR circuit, visualized with immunostaining for cFos (red), just before being killed. $\boldsymbol{a}-\boldsymbol{i}$, Representative images of transverse, L6 spinal cord sections are shown. There were more total $\mathrm{F}$. ${ }^{+}{ }^{+}$neurons and PRV ${ }^{+}$ neurons in lumbar spinal cords of T3Tx-Saline animals than in T3Tx-XPro1595 $(\boldsymbol{a}, \boldsymbol{b}, \boldsymbol{d}, \boldsymbol{e}, \boldsymbol{g}, \boldsymbol{h})$. There were also fewer sympathetically associated neurons activated (Figure legend continues.) 
through the $24 \mathrm{~h}$ recordings for paroxysmal hypertension and reflexive bradycardia (Fig. $3 h-k$ ). Both T3Tx-Saline and T3TxXPro1595 animals had similar numbers of events at 2 weeks (Fig. $3 j ; p=0.1468)$. AD in the T3Tx-Saline animals intensified over time - the number of detected events increased between 2 and 8 weeks post-SCI (Fig. 3j; $F_{(1,156)}=20.37, p<0.0001$, post hoc, $p=$ 0.0089 ), corroborating the progressive intensification of $\mathrm{AD}$ over time described previously (Krassioukov and Weaver, 1995; Zhang et al., 2013; West et al., 2015). In contrast, there was no change in the frequency of AD events in T3Tx-XPro1595 animals over time. Additionally, T3Tx-XPro1595 animals had significantly fewer naturally occurring events at 4,6 , and 8 weeks postSCI (Fig. 3j; $F_{(1,156)}=20.37, p<0.0001$, post hoc, at 4 weeks $p=$ 0.0366 , post hoc, at 6 weeks $p=0.0107$, post hoc, at 8 weeks $p=$ $0.0046)$. We also found that T3Tx-Saline animals had significantly greater peak MAP per AD event at each time point (Fig. $3 k$; $F_{(1,481)}=52.01, p<0.0001$, post hoc, at 2 weeks $p=0.0229$, at 4 weeks $p=0.0162$, at 6 weeks $p<0.0001$, at 8 weeks $p=0.0165$ ). Collectively, these data indicate that inhibiting spinal sTNF $\alpha$ starting $3 \mathrm{~d}$ after injury below SCI attenuates the severity and progressive exacerbation of $\mathrm{AD}$.

\section{Delayed spinal sTNF $\alpha$ inhibition improves splenic leukocyte profile after complete high thoracic SCI}

People with SCI often have compromised immune systems and are at higher risk for infection (Failli et al., 2012). Moreover, those with high-level injuries who experience $\mathrm{AD}$, indicative of heightened SSR circuit activation and sympathetic hyperreflexia, are at even higher risk of infection (Zhang et al., 2013; Brommer et al., 2016). This is likely a consequence of increased sympathetic activity in immune-related effector organs, such as the spleen. Indeed, recurrent activation of the SSR circuit after high-level SCI results in splenic atrophy and diminished B cell numbers (Zhang et al., 2013). Because XPro1595 treatment attenuates AD development, suggestive of diminished activation of the SSR circuit, we wanted to determine whether XPro1595 treatment also attenuates peripheral immune dysfunction resultant from sympathetic hyperreflexia (Zhang et al., 2013; Ueno et al., 2016).

We first evaluated leukocyte and lymphocyte levels within the spleen, a major secondary lymphoid organ involved in regulating the immune system that receives sympathetic input. Spleens from uninjured animals, T3Tx-Saline and -XPro1595 animals 8 weeks post-SCI were harvested and splenocytes were counted and characterized via flow cytometry.

As reported previously, chronic T3Tx results in dramatic splenic atrophy. We observed that T3Tx-Saline animals had smaller spleens (Fig. $4 a, b ; F_{(2,14)}=6.306, p=0.0112$, post hoc, vs naive $p=0.0137$ ) and fewer total splenocyte numbers than naive animals (Fig. $4 c ; F_{(2,14)}=5.239, p=0.0199$, post hoc, vs naive $p=$ $0.0201)$. Treatment with XPro1595 prevented the SCI-induced splenic atrophy; T3Tx-XPro1595 animals had spleen weights that were higher than T3Tx-Saline animals and were similar to naive

\section{$\leftarrow$}

(Figure legend continued.) by (RD (i.e., $\mathrm{CFos}^{+} / \mathrm{PRV}^{+}$) in T3Tx-Saline animals than T3TxXPro1595 animals $(\boldsymbol{c}, \boldsymbol{f}, \boldsymbol{i}) . \boldsymbol{j}-\boldsymbol{t}$, Representative images of the IML in longitudinal, T8-T13 spinal cord sections are shown. While there was no difference in the number of SPNs within the SSR circuit that were activated by $\mathrm{CRD}$ (i.e., PRV ${ }^{+} / \mathrm{CFos}^{+} / \mathrm{FG}^{+}$; see $\boldsymbol{m}^{\prime \prime}$ ) between the two groups $(\boldsymbol{j}-\boldsymbol{q}, \boldsymbol{s})$, there were more sympathetically associated interneurons that were activated by CRD (i.e., PRV ${ }^{+} / \mathrm{CFOS}^{+} / \mathrm{FG}^{-}$cells; see $\boldsymbol{m}^{\prime}$ ) in T3TX-Saline animals $(\boldsymbol{j}-\boldsymbol{m})$ than in T3TX-XPro1595 animals $(\boldsymbol{n}-\boldsymbol{r})$. As a result, the ratio of CRD-responsive interneurons to SPNs within the SSR circuit in T3TX-XPro1595 animals was less than in saline-treated animals $(\boldsymbol{t}) . n=4$ /group. Mean \pm SEM ${ }^{*} p<0.05 ;{ }^{* *} p<0.01 ;{ }^{* * *} p<0.001$. Scale bars, $100 \mu \mathrm{m}$. animals (Fig. $4 a, b ; F_{(2,14)}=6.306, p=0.0112$, post hoc, vs T3TxSaline $p=0.0059)$. T3Tx-XPro1595 animals also had normal (i.e., similar to naive) numbers of splenocytes that were higher than T3Tx-Saline (Fig. $4 c ; F_{(2,14)}=5.239, p=0.0199$, post hoc, vs T3Tx-Saline $p=0.0111$ ).

We also found striking differences in the profile of the splenocytes, as determined by flow cytometry (the gating strategy is depicted in Fig. 4d). T3Tx-XPro1595 animals had more CD68 ${ }^{+}$ monocytes compared with uninjured animals (Fig. $4 e ; F_{(2,14)}=$ $2.468, p=0.1208$, post hoc, vs naive $p=0.0492$ ). Corresponding to the splenocyte count numbers in Figure $4 c$, T3Tx-Saline animals had fewer CD45R ${ }^{+}$B cells (Fig. $4 f ; F_{(2,14)}=4.045, p=$ 0.0411 , post hoc, vs naive $p=0.0405$, vs T3Tx-XPro1595 $p=$ 0.0211 ), $\mathrm{CD}^{+} \mathrm{T}$ cells (Fig. $4 g ; F_{(2,14)}=7.5, p=0.0061$, post hoc, vs naive $p=0.0077$, vs T3Tx-XPro1595 $p=0.0034), \mathrm{CD}^{+} \mathrm{T}$ cells (Fig. $4 h ; F_{(2,14)}=10.55, p=0.0016$, post hoc, vs naive $p=$ 0.0381 , vs T3Tx-XPro1595 $p=0.0004$ ), and CD ${ }^{+}$T cells (Fig. $4 i ; F_{(2,14)}=6.083, p=0.0065$, post hoc, vs naive $p=0.0015$, vs T3Tx-XPro1595 $p=0.0388$ ) than either naive or T3TxXPro1595 animals. Notably, numbers of CD45R ${ }^{+}$B cells, CD3 ${ }^{+}$ $\mathrm{T}$ cells, $\mathrm{CD}^{+}{ }^{+} \mathrm{T}$ cells, and CD4 ${ }^{+} \mathrm{T}$ cells in T3Tx-XPro1595 spleens were similar to those seen in naive animals. Last, we found that T3Tx-XPro1595 animals had more CD4 ${ }^{+} / \mathrm{FoxP}^{+} \mathrm{T}_{\mathrm{REG}}$ cells than either naive or T3Tx-Saline animals (Fig. $4 j ; F_{(2,14)}=$ $6.312, p=0.0111$, post hoc, vs naive $p=0.0434$, vs T3Tx-Saline $p=0.0036)$.

Collectively, these data indicate that SCI detrimentally alters the profile of splenocytes in a way suggestive of a compromised immune system. Moreover, inhibiting sTNF $\alpha$ centrally with XPro1595 attenuates these changes, implying improved immune function.

\section{Intrathecal administration of XPro1595 does not affect splenic corticosterone levels but does diminish the surge in $\mathrm{NE}$ expression and sprouting of adrenergic fibers within the spleen after SCI}

Increased glucocorticoid and noradrenergic signaling within the spleen have been implicated in the decrease in splenocyte number after high-level SCI (Lucin et al., 2009; Zhang et al., 2013), such as what we saw in our studies here (Fig. 4). Does intrathecal administration of XPro1595 diminish levels of corticosterone (CORT) or norepinephrine (NE) in the spleen? We found that both T3TxSaline and -XPro1595 treated animals 8 weeks after SCI had splenic CORT levels that strongly trended higher than that in spleens from uninjured animals. There was no difference in CORT levels between the T3Tx groups (Fig. $5 a$; naive vs T3TxSaline $p=0.0607$, naive vs T3Tx-XPro1595 $p=0.0656$ ). However, we did see a treatment effect in splenic NE levels. Spleens from T3Tx-Saline animals had higher levels of NE than spleens from either uninjured or T3Tx-XPro1595 animals (Fig. 5b; $F_{(2,11)}$ $=10.17, p=0.0032$, post hoc, vs naive $p=0.0014$, vs T3TxXPro1595 $p=0.0062$ ). T3Tx-XPro1595 animals had similar NE levels to naive animals. We also found that the extent of $\mathrm{TH}^{+}$, sympathetic postganglionic fibers innervating the spleen correlated with NE levels. T3Tx-Saline animals had more $\mathrm{TH}^{+}$fibers in lymphocyte-rich, $\mathrm{CD} 45^{+}$white pulp regions than naive or T3Tx-XPro1595 animals (Fig. $5 c-m ; F_{(2,39)}=5.897, p=0.0058$, post hoc, vs naive $p=0.0024$; vs T3Tx-XPro1595 $p=0.013$ ). Furthermore, the $\mathrm{CD} 45^{+}$regions were smaller in the T3TxSaline animals (Fig. $5 l ; F_{(2,39)}=6.314, p=0.0042$, post hoc, vs naive $p=0.0011$; vs T3T $x$-XPro1595 $p=0.0337$ ), corroborating the loss of lymphocytes observed in our flow analyses (Fig. 4). T3Tx-XPro1595 and uninjured animals had similar $\mathrm{TH}^{+}$immunoreactivity in similarly sized $\mathrm{CD}_{4}{ }^{+}$white pulp regions 
a Baseline Mean Arterial Pressure

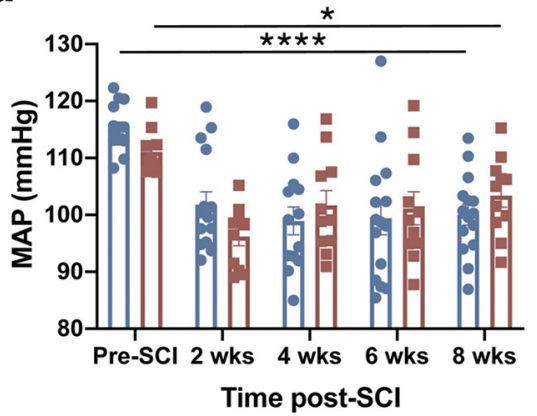

b

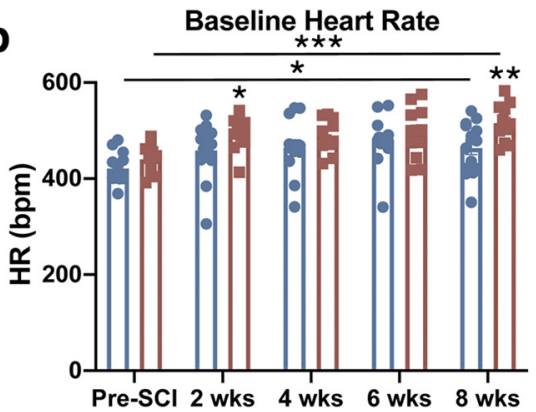

Time post-SCI
C

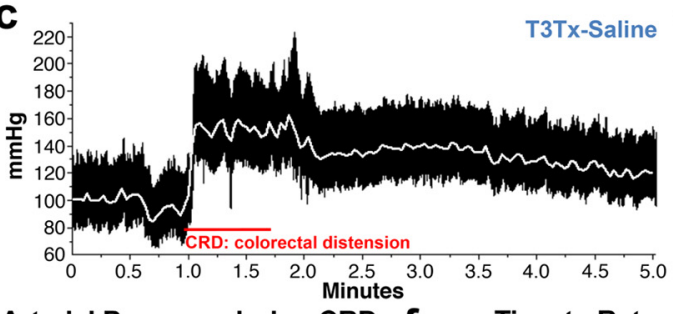

d

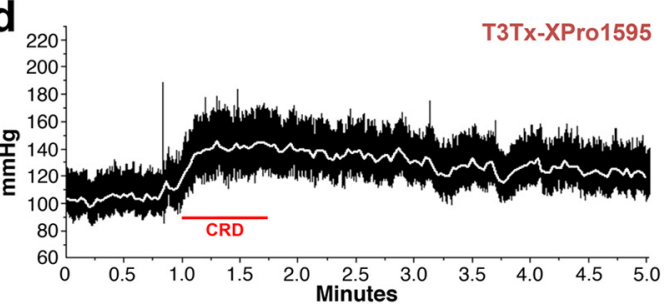

$\Delta$ Mean Arterial Pressure during CRD $f$ Time to Return to Baseline MAP

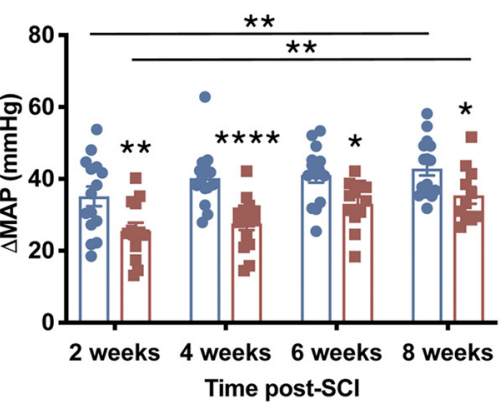

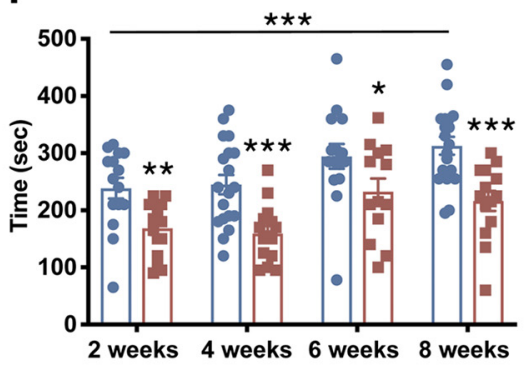

Time post-SCl

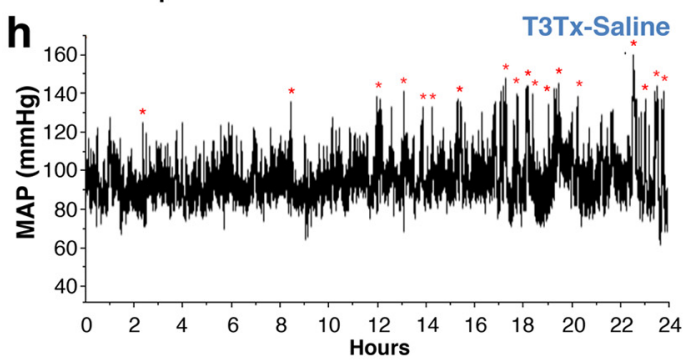

i

g

$\Delta$ Heart Rate during CRD

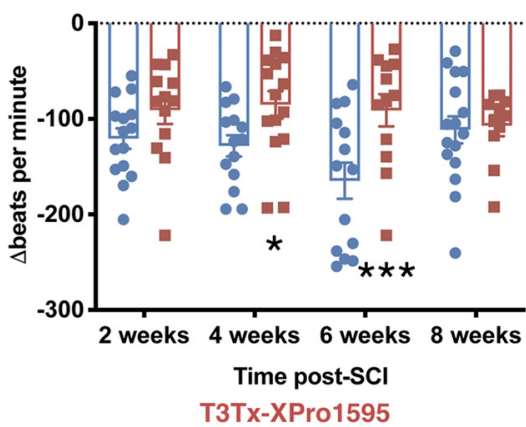

T3Tx-XPro1595
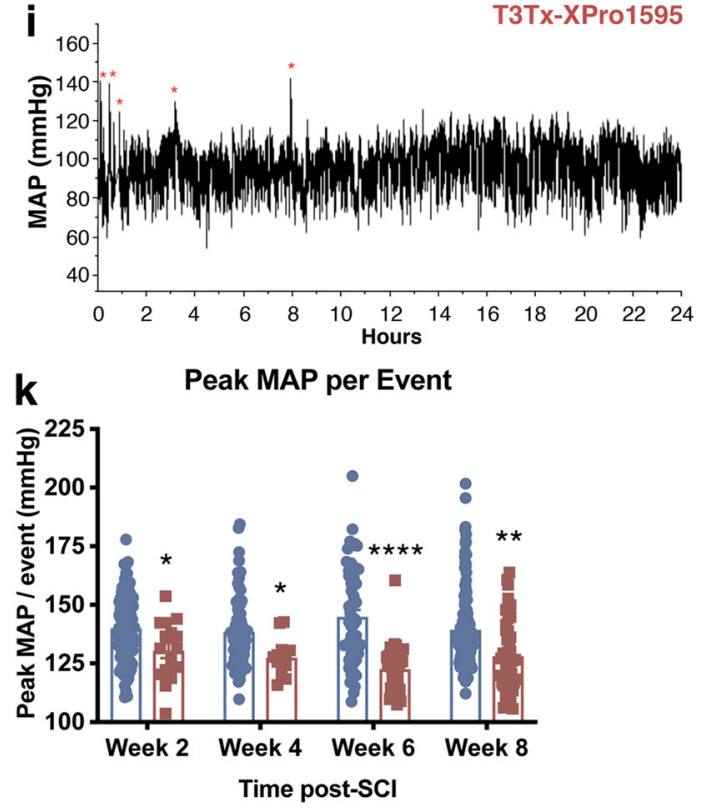

T3Tx-Saline

Figure 3. XPro1595 treatment initiated $3 \mathrm{~d}$ post-injury is sufficient to attenuate autonomic dysreflexia, a gauge of sympathetic hyperreflexia. $\boldsymbol{a}, \boldsymbol{b}$, Baseline mean arterial pressure (MAP) and heart rate (HR) were assessed preinjury and at biweekly time points after T3Tx. In both groups, the injury itself resulted in a persistent decrease in MAP. There was no difference in MAP between T3TX animals treated with saline or XPro1595 (a). Injury also resulted in higher HR in both groups. At 2 and 8 weeks post-T3Tx, XPro1595 animals had faster HR than saline treated animals $(\boldsymbol{b})$. $\boldsymbol{c}-\boldsymbol{g}$, AD events were experimentally induced via $1 \mathrm{~min}$ of colorectal distension (CRD). Representative beat-to-beat arterial traces from (c) T3Tx-Saline animals and (d) T3Tx-XPro1595 treated animals 8 weeks after injury are shown. The white line indicates MAP. Although both groups exhibit a sharp increase in MAP in response to CRD, T3TX-Saline animals had far greater responses than T3Tx-XPro1595 animals at every time point (e) that took longer to return to baseline MAP after CRD ended ( $\boldsymbol{f}$ ). In both T3TX-Saline and -XPro1595 animals, the CRD-induced $\triangle$ MAP increased over time, suggesting that AD progressively worsens (e). T3TX-XPro1595 animals also exhibit less reflexive bradycardia than T3TX-saline animals at 46 -weeks post-injury $(\boldsymbol{f}) . \boldsymbol{h}-\boldsymbol{k}$, Continuous $24 \mathrm{~h}$ hemodynamic recordings were conducted to assess naturally occurring AD events. Representative MAP traces from T3TX-Saline ( $h$ ) or T3TX-XPro1595 (i) animals over $24 \mathrm{~h}$ at 8 weeks post-SCl are shown. Naturally occurring AD episodes are indicated by red asterisks. XPro1595-treated animals exhibit fewer daily naturally occurring AD events compared with saline controls at 4, 6, (Figure legend continues.) 

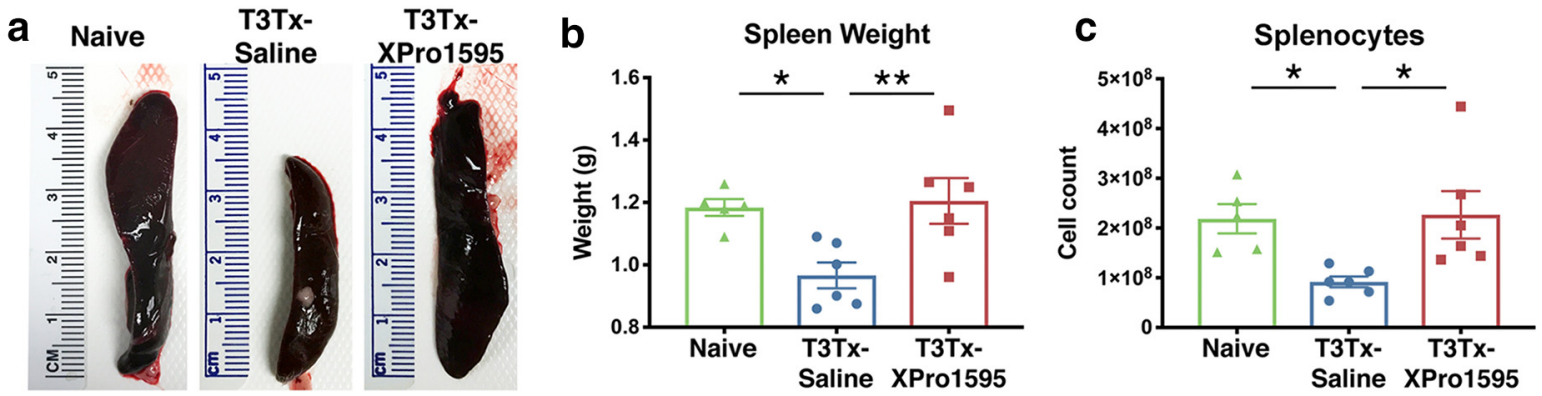

d

Gating: Singlets

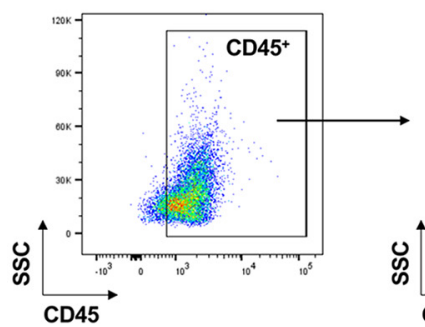

Gating: $\mathrm{CD}^{4+} 5^{+}$leukocytes
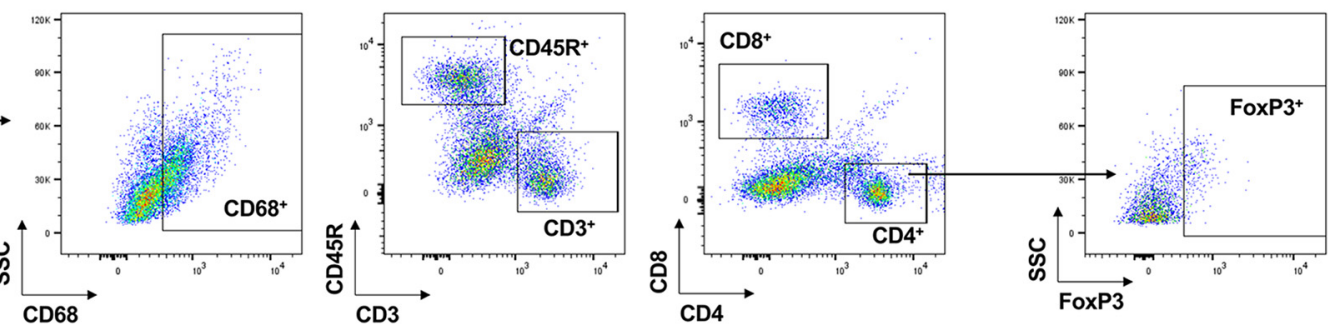

e

$\mathrm{CD}_{68}^{+} \mathrm{M} \theta$

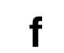

CD45R ${ }^{+}$B cells
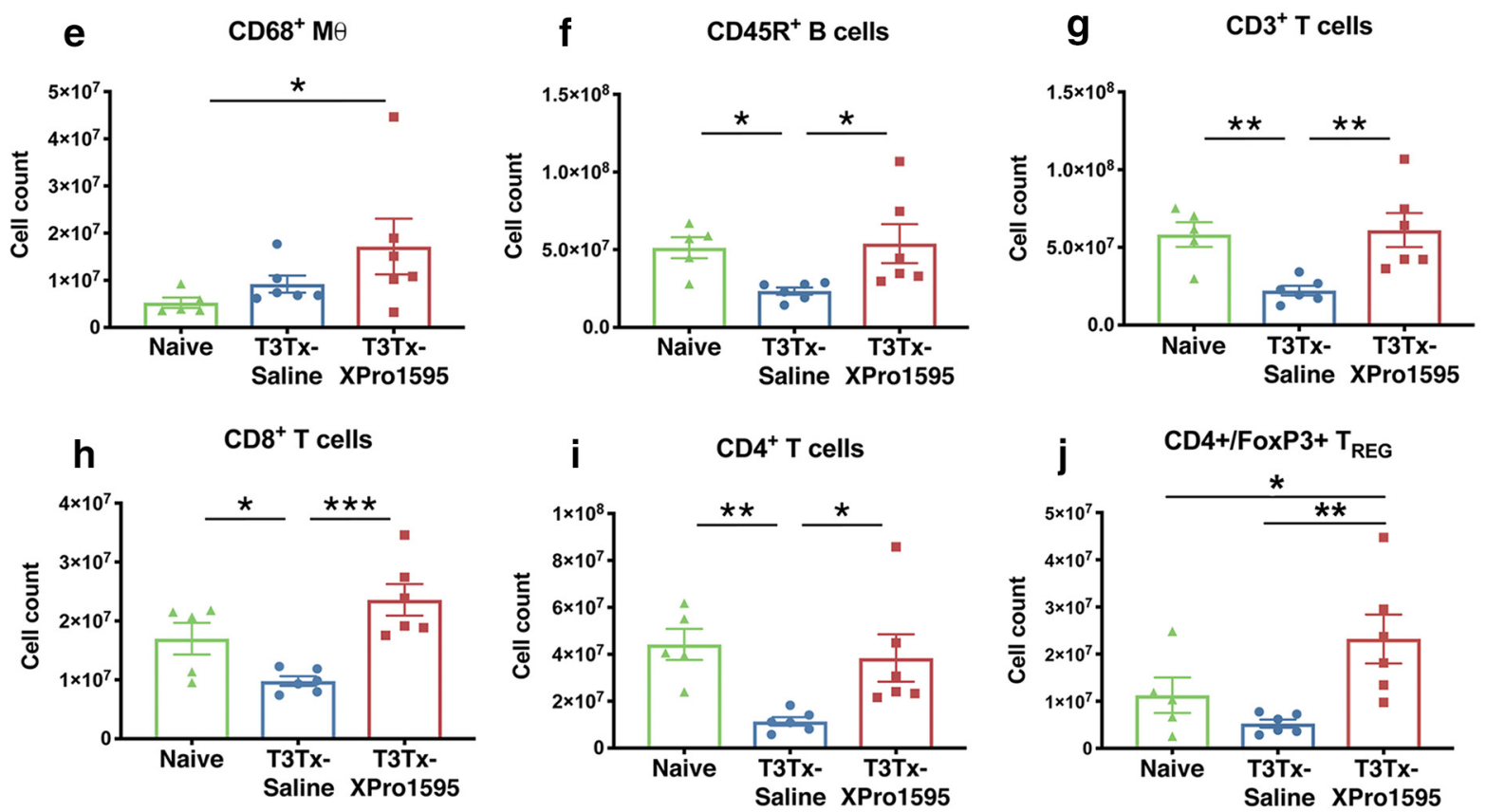

Figure 4. XPro1595-treated animals exhibit more normal splenic leukocyte profiles. Spleens of T3TX-Saline and-XPro1595 animals were harvested 8 weeks post-SCl and compared with those of naive, uninjured animals. $\boldsymbol{a}, \boldsymbol{b}$, Spleens from T3TX-Saline underwent dramatic atrophy and were smaller than spleens from naive and T3Tx-XPro1595 animals. In contrast, spleens from T3TXXPro1595 animals had similar weights to those in naive animals. $\mathbf{c}-\boldsymbol{j}$, Lymphocyte cell numbers were determined via flow cytometry. The gating strategy is shown in (d). There was an overall decrease in splenocyte number in T3TX-Saline animals only (c), corresponding with the splenic atrophy in these animals. T3Tx-XPro1595 animals had more total monocyte-derived CD68 ${ }^{+}$ macrophages than naive animals while T3Tx-Saline animals had similar numbers of $\mathrm{CD68}{ }^{+}$macrophages $(\boldsymbol{e})$. T3Tx-Saline animals also had fewer total CD45R ${ }^{+} \mathrm{B}$ cells, $\mathrm{CD} 3{ }^{+} \mathrm{T}$ cells, $\mathrm{CD} 8{ }^{+} \mathrm{T}$ cells, and $\mathrm{CD} 4{ }^{+} \mathrm{T}$ cells than naive and XPro1595-treated animals $(\boldsymbol{f}-\boldsymbol{i})$. The number of $\mathrm{CD} 4^{+} / \mathrm{FoxP3}{ }^{+}$regulatory $\mathrm{T}$ cells in T3TX-Saline animals was similar to that in naive animals while $\mathrm{T3Tx}$-XPro 1595 animals had more $\mathrm{T}_{\text {Regs }}$ than both naive and saline-treated animals $(j) . n=5$-6/group. Mean $\pm S E M{ }^{*} p<0.05 ;{ }^{* *} p<0.01 ;{ }^{* * *} p<0.001$.

$\leftarrow$

(Figure legend continued.) and 8 weeks post-SCI (j). The number of events increased over time in T3TX-Saline animals but not XPro1595-treated animals between 2- and 8-weeks (j). The peak MAP per event was lower in T3Tx-XPro1595 animals compared with saline-treated controls $(\boldsymbol{k}) . n=18-24$ /group. Mean \pm SEM ${ }^{*} p<0.05 ;{ }^{* *} p<0.01 ;{ }^{* * *} p<0.001 ;{ }^{* * * *} p<$ 0.0001 .
(Fig. $5 c-m$ ), again correlating with our flow and NE ELISA data. These data suggest that intrathecal XPro1595 prevents splenic atrophy after SCI via a CORT-independent, NE-dependent mechanism.

Delayed intrathecal XPro1595 treatment improves immunity against bacterial pneumonia after chronic SCI

We wanted to more directly assess whether T3Tx-XPro1595 animals had improved immunity against infection. As respiratory 
a

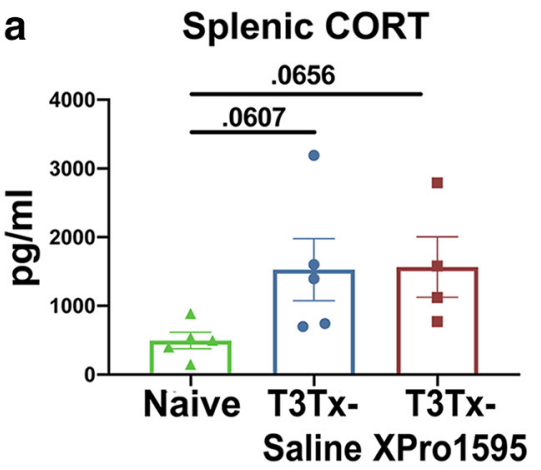

b

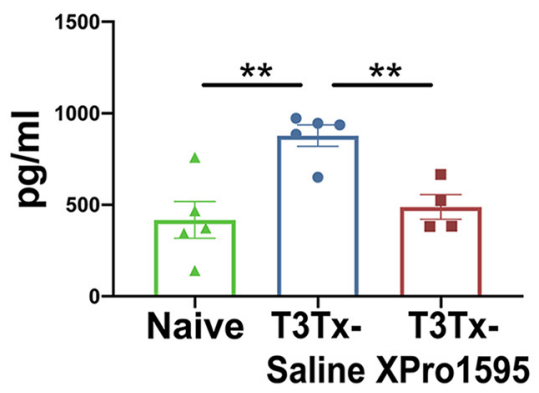

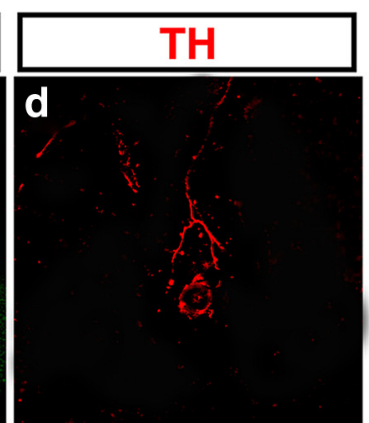
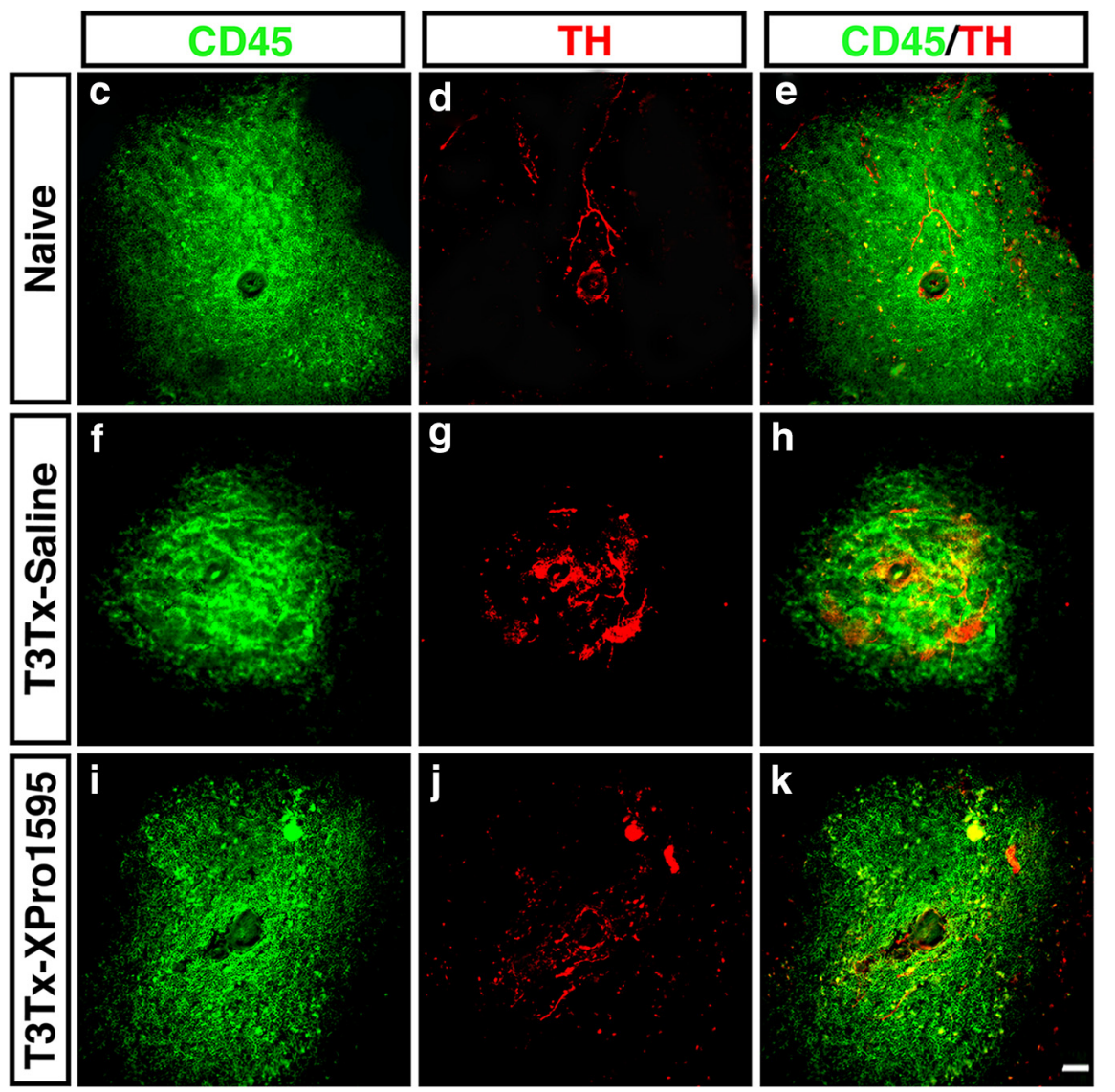

CD45+ area

I

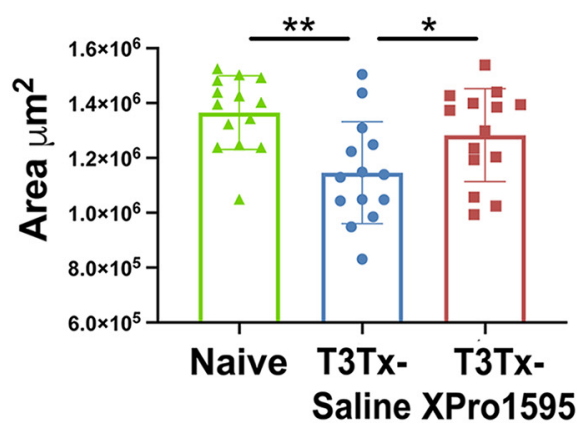

m

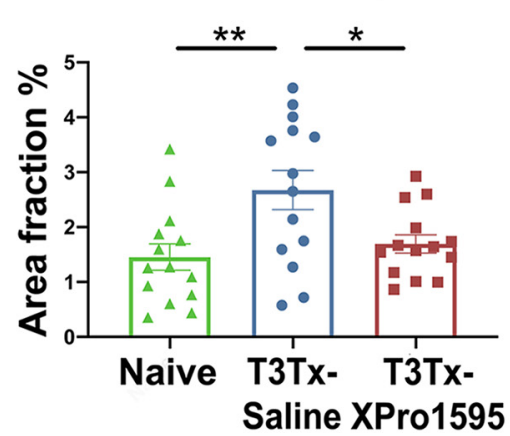

Figure 5. Intrathecal administration of XPro1595 does not affect splenic corticosterone levels but does diminish the surge in NE expression and sprouting of adrenergic fibers within the spleen after $\mathrm{SCl} . \boldsymbol{a}, \boldsymbol{b}$, Spleens were harvested 8 weeks after $\mathrm{SCl}$ and assessed for corticosterone (CORT) or norepinephrine (NE) levels via ELISA. Spleens from naive animals had a basal level of CORT and NE. Both T3TX-Saline animals and T3Tx-XPro1595 exhibited a strong trend toward increased splenic CORT. T3Tx-Saline animals had greater levels of splenic NE than naive or T3Tx-XPr01595 animals. $\mathbf{c} \boldsymbol{- m}$, Transverse sections of spleens from naive, uninjured animals or T3TX-Saline or -XPr01595 animals 8 weeks after SCI were immunostained for CD45 (green) to label leukocytes in white pulp regions and $\mathrm{TH}$ (red) to identify adrenergic fibers. Spleens from naive animals had large $\mathrm{CD}_{4}{ }^{+}$islands (c) innervated by $\mathrm{TH}^{+}$

infections (e.g., pneumonia) are prevalent in the SCI population, we were specifically interested in how animals with T3Tx respond to infection with the bacteria $S$. pneumoniae. T3Tx-Saline and -XPro1595 animals were infected with $S$. pneumoniae intratracheally at 8 weeks post-SCI. Agematched, uninjured animals were infected similarly. No animals were administered antibiotics and all animals were monitored daily for $10 \mathrm{~d}$ postinfection.

Although no uninjured animals died after S. pneumoniae infection (Fig. 6a), they all became ill. They lost a significant amount of weight (Fig. 6b), had high body temperatures (Fig. 6c), and exhibited tachypnea (Fig. $6 d$ ). All uninjured, infected animals recovered by $8 \mathrm{~d}$, as indicated by a return of body weight, body temperature, and respiratory rate to baseline levels (Fig. $6 b-d$; Table 2). On the contrary, 3 of 8 T3Tx-Saline animals (37.5\%) died within $5 \mathrm{~d}$ after infection (Fig. 6a). The T3Tx-Saline animals that survived the entire 10-day assessment period had persistent weight loss (Fig. 6b). Additionally, these animals had higher body temperatures (Fig. $6 c$ ) and respiratory rates early on (Fig. $6 d$ ), indicating that they were sicker than the uninjured animals (Table 2). Remarkably, intrathecal administration of XPro1595 improved immunity against $S$. pneumoniae infection. While infected, uninjured and infected, T3Tx-XPro1595 animals had similar elevations in body temperature (Fig. 6c), T3Tx-XPro1595 animals did lose more weight (Fig. 6b) and displayed increased respiratory rate (Fig. $6 d$ ), indicating that T3Tx-XPro1595 animals got sicker. However, unlike T3Tx-Saline animals, all infected, T3Tx-XPro1595 animals survived (Fig. 6a) and returned to baseline parameters by $10 \mathrm{~d}$ postinfection (Fig. 6b-d).

To determine how well the immune system was able to clear the bacteria, lungs were harvested from all surviving animals $10 \mathrm{~d}$ postinfection, homogenized, and cultured on blood agar plates for $48 \mathrm{~h}$. Lungs from uninjured, uninfected animals were also harvested for comparison.

\footnotetext{
axons $(\boldsymbol{d}, \boldsymbol{e})$. T3Tx-Saline animals had smaller $\mathrm{CD} 45^{+}$white pulp regions than naive animals $(\boldsymbol{f})$, that were innervated by more $\mathrm{TH}^{+}$fibers $(\boldsymbol{g}, \boldsymbol{I}, \boldsymbol{m})$. CD45 ${ }^{+}$white pulp in T3TXXPro1595 spleens were similar in size to naive animals and were larger than those in saline-treated animals $(\boldsymbol{i}, \boldsymbol{l})$. Moreover, $\mathrm{TH}^{+}$immunostaining in white pulp in T3TX-XPro1595 animals was similar to naive animals and was less than that in T3TX-Saline animals $(\boldsymbol{j}, \boldsymbol{m}) . n=4-5 /$ group. Mean \pm SEM ${ }^{*} p<0.05 ;{ }^{* *} p<0.01$. Scale bar, $50 \mu \mathrm{m}$.
} 


\section{- Naive - Uninjured + Infection - T3Tx-Saline + Infection + T3Tx-XPro1595 + Infection}

a

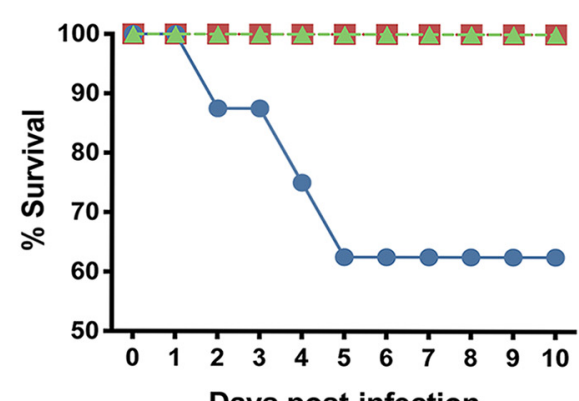

C

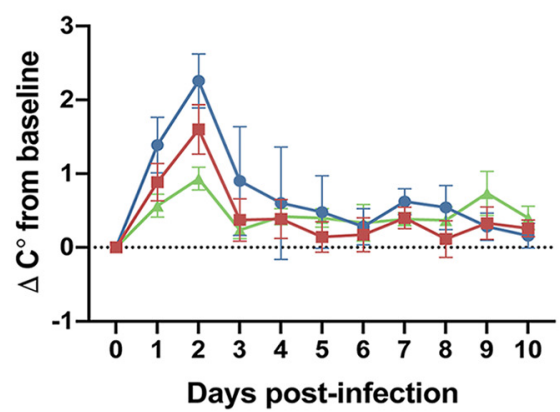

b

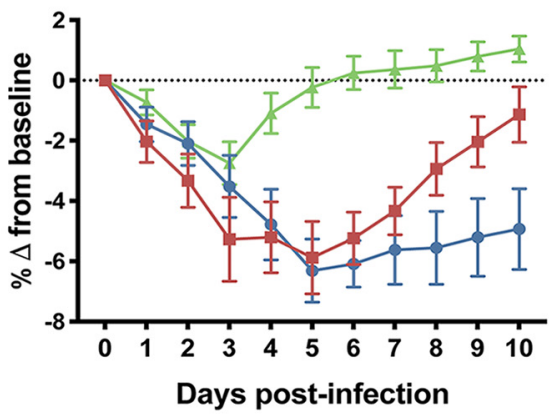

d

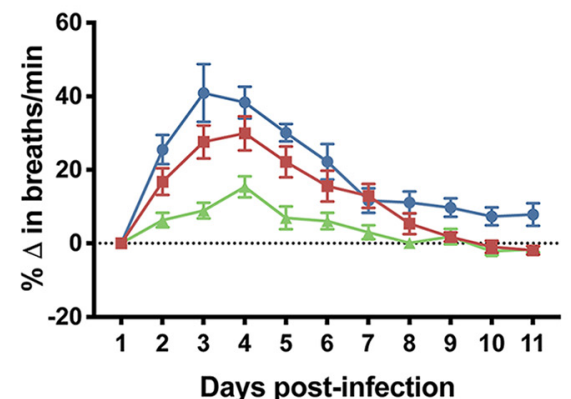

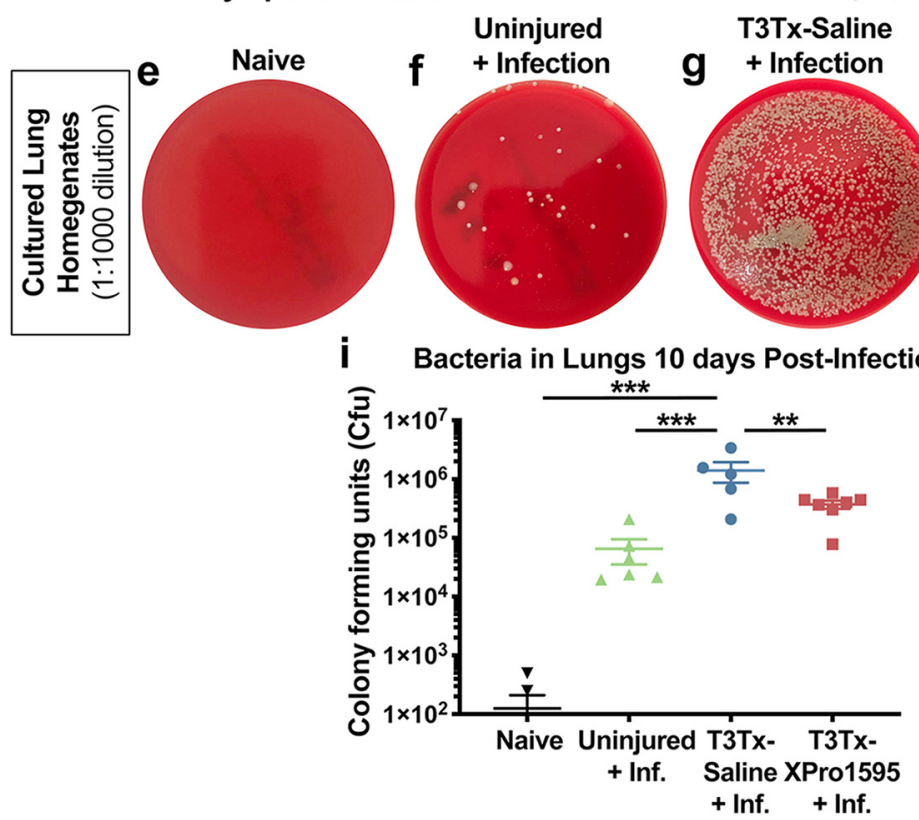

Figure 6. Delayed intrathecal XPro1595 treatment after T3Tx improves antibacterial immunity in chronic SCI animals. At 8 weeks post-SCI, T3Tx-Saline and T3Tx-XPro1595 animals were intracheally administered S. pneumoniae and sickness behavior was tracked for $10 \mathrm{~d}$. Uninjured animals were similarly infected for comparison. $\boldsymbol{a}$, After infection, $37.5 \%$ ( $3 / 8$ ) of T3Tx-Saline animals died. No uninjured (0/6) or T3Tx-XPro1595 (0/7) animals died after infection - these latter groups had a 100\% survival rate. $\boldsymbol{b}$ - $\boldsymbol{d}$, Uninjured, infected animals exhibited decreased body weight, increased body temperature, and increased respiratory rate, indicative of sickness. T3Tx-Saline animals exhibited an even greater degree of weight loss, fever, and tachypnea than uninjured and T3TX-XPro1595 animals. T3TX-Saline animals were unable to recover to normal bodyweight $(\boldsymbol{b})$ but recovered back to normal body temperature (c) and respiratory rate (d). T3Tx-XPro1595 animals also exhibited sickness behavior but they recovered to normal bodyweight $(\boldsymbol{b})$ and did not have as high a peak body temperature $(\boldsymbol{c})$ or respiratory rate $(\boldsymbol{d})$. $\boldsymbol{e}-\boldsymbol{i}$, Lungs were harvested $10 \mathrm{~d}$ after infection and homogenates were plated onto blood agar plates. Lungs from naive (i.e., uninfected, uninjured) animals were also harvested and cultured as an additional control. Colony forming units (CFU) were counted $48 \mathrm{~h}$ later. There were some CFU in lung cultures from infected, uninjured animals, suggesting that $S$. pneumoniae was not completely cleared from the lungs ( $\boldsymbol{f}, \boldsymbol{i})$. Cultures from T3Tx-Saline animals' lungs $(\boldsymbol{g})$ had more CFUs than those from uninjured-infected animals $(\boldsymbol{f})$, indicating that T3Tx reduces bacterial clearance, indicative of diminished immune function. Cultures from XPro1595-treated animals $(\boldsymbol{h})$ had fewer CFUs than saline-treated animals and were similar to uninjured-infected animals $(\boldsymbol{i}) . n=5-7 /$ group. Mean \pm SEM ${ }^{* *} p<0.01$; ${ }^{* * *} p<0.001$.

There were some colonies in the cultures using lungs from infected, uninjured animals (Fig. 6f,i), indicating there was some bacteria present in these animals' lungs at the time of harvest. There were many more colonies in the cultures from T3Tx-Saline animals, indicating that there was still a significant bacterial presence in their lungs (Fig. $6 g, i ; F_{(3,20)}=7.366, p=0.0016$, post hoc, vs naive $p=0.0004$, vs uninjured-infected $p=0.0007$ ). Cultures from T3Tx-XPro1595 animals had fewer colonies than those 
Table 2. Comparison of outcomes over $10 \mathrm{~d}$ after S. pneumoniae infection

\begin{tabular}{|c|c|c|c|}
\hline Experimental group & Body weight & Temperature & Respiratory rate \\
\hline \multirow[t]{3}{*}{ Uninjured-infected over time (compared with pre-infection baseline) } & ${ }^{*} p=0.0147$ at day 1 & ${ }^{*} p<0.05$ at days $1,4-5$ & ${ }^{*} p<0.05$ at days 1,5 \\
\hline & ${ }^{*} p=0.0116$ at day 2 & ${ }^{* *} p<0.01$ at days 2,7 & ${ }^{* *} p<0.01$ at days $2-3$ \\
\hline & NS at days $3-10$ & NS at days $3,8-10$ & NS at days $4,6-10$ \\
\hline \multirow[t]{4}{*}{ T3Tx-Saline + infection over time (compared with pre-infection baseline) } & ${ }^{*} p<0.05$ at days $2-10$ & ${ }^{* *} p=0.0078$ at day 1 & ${ }^{* *} p<0.01$ at days $1-2$ \\
\hline & & $* * * p=0.0007$ at day 2 & $* * * p<0.001$ at days $3-4$ \\
\hline & & ${ }^{*} p=0.0169$ at day 7 & ${ }^{*} p<0.05$ at days $5-9$ \\
\hline & & NS at days $3-6,8-10$ & NS at day 10 \\
\hline \multirow[t]{4}{*}{ T3Tx-XPro1595 + infection over time (compared with pre-infection baseline) } & ${ }^{*} p<0.05$ at day $1-9$ & ${ }^{*} p<0.05$ at days 1,7 & ${ }^{* *} p<0.01$ at days $1,4,6$ \\
\hline & NS at day 10 & ${ }^{* *} p=0.0031$ at day 2 & ${ }^{* * *} p<0.001$ at days $2-3$ \\
\hline & & NS at days $3-6,8-10$ & ${ }^{*} p=0.0101$ at day 5 \\
\hline & & & NS at days $7-10$ \\
\hline \multirow[t]{5}{*}{ T3TX-Saline + infection versus uninjured-infected } & NS at days $0-3$ & ${ }^{*} p=0.0384$ at day 2 & ${ }^{* * *} p=0.0001$ at day 1 \\
\hline & ${ }^{*} p=0.0117$ at day 4 & NS at days $1,3-10$ & ${ }^{* * * *} p<0.0001$ at days $2-4$ \\
\hline & $* * * * p<0.0001$ at days $5-10$ & & ${ }^{* *} p=0.0015$ at day 5 \\
\hline & & & NS at days $6,8-10$ \\
\hline & & & ${ }^{*} p=0.0454$ at day 7 \\
\hline \multirow[t]{6}{*}{ T3Tx-XPro1595 + infection versus Uninjured-infected } & NS at days $0-3$ & NS at days $1-10$ & ${ }^{*} p=0.0367$ at day 1 \\
\hline & ${ }^{* *} p=0.0016$ at day 4 & & ${ }^{* * * *} p<0.0001$ at day 2 \\
\hline & ${ }^{* * * *} p<0.0001$ at days $5-6$ & & ${ }^{* *} p<0.01$ at days $3-4$ \\
\hline & $* * * p=0.0003$ at day 7 & & NS at days $5-10$ \\
\hline & ${ }^{*} p<0.05$ at days $8-9$ & & \\
\hline & NS at day 10 & & \\
\hline \multirow[t]{3}{*}{ T3TX-Saline + infection versus T3Tx-XPro1595 + infection } & NS at day $0-8$ & NS at days $1-10$ & NS at days 1, 3-10 \\
\hline & ${ }^{*} p=0.0293$ at day 9 & & ${ }^{* *} p=0.0085$ at day 2 \\
\hline & ${ }^{* *} p=0.0067$ at day 10 & & \\
\hline
\end{tabular}

NS, nonsignificant.

from T3Tx-Saline animals and were similar to both uninjured groups, regardless of whether they were infected (Fig. $6 h, i ; F_{(3,20)}$ $=7.366, p=0.0016$, post hoc, vs naive $p=0.2374$, vs uninjuredinfected $p=0.3266$, vs T3Tx-Saline, $p=0.0044)$. These data indicate that inhibiting sTNF $\alpha$ centrally post-SCI dramatically attenuates SCI-induced immunosuppression and improves immunity in chronic SCI animals.

\section{Discussion}

Regulation of sympathetic activity is critical for maintaining normal function of organs that receive sympathetic input. Thus, it is not surprising that organ dysfunction commonly occurs after SCI (Alan et al., 2010; Zhang et al., 2013; Sauerbeck et al., 2015; Kigerl et al., 2016; Ueno et al., 2016; Prüss et al., 2017). These detrimental secondary, systemic consequences of SCI are even more severe with high-level injuries (Alan et al., 2010; Zhang et al., 2013; Sauerbeck et al., 2015; Ueno et al., 2016; Prüss et al., 2017), suggesting that loss of descending modulation of spinal sympathetic output plays a major role. Furthermore, considerable evidence suggests that SCI induces intraspinal plasticity that renders SSR circuitry more excitable. This results in sympathetic hyperreflexia that manifests as $\mathrm{AD}$ and drives immunodeficiency (Weaver et al., 2001; Prüss et al., 2017). The mechanisms by which SSR circuits become hyperexcitable after injury remain elusive. Additionally, the degree to which plasticity of SSR circuits causes secondary consequences of SCI (e.g., AD and dysimmunity) is also poorly understood. We surmised that persistent neuroimmune signaling below an injury influences activity of the SSR circuit to profoundly impact the organs innervated by the sympathetic nervous system. Here, we set out to determine whether administering XPro1595 to inhibit sTNF $\alpha$ beginning at a clinically feasible, post-SCI time point ( $3 \mathrm{~d}$ ) could sufficiently blunt plasticity of the SSR circuit to therapeutically hamper AD development and improve immunity months after SCI. Furthermore, we also wanted to assess whether intrathecal XPro1595 treatment improved chronic SCI animals' immunity sufficiently enough to fend off a clinically relevant $S$. pneumoniae infection challenge.

We found that delayed sTNF $\alpha$ inhibition decreased the severity of $\mathrm{AD}$ when compared with the saline-treated controls (Fig. 3 ), similar to what we saw when XPro1595 was administered just after SCI (Mironets et al., 2018), indicating that sTNF $\alpha$-mediated SSR circuitry plasticity occurs or becomes functional at some point after $3 \mathrm{~d}$. This bolsters the notion of XPro1595 as a prophylactic treatment for this life-threatening syndrome, a gauge of sympathetic hyperreflexia. However, unlike what we saw previously, we also observed that CRD-induced AD intensified between 2 and 8 weeks (West et al., 2016) post-T3Tx, even with XPro1595 treatment, albeit to a far lesser degree that salinetreated animals (Fig. 3c). This suggests that factors other than sTNF $\alpha$ signaling play a role in AD development. Future work will identify mechanisms independent of sTNF $\alpha$ that potentiate AD.

Importantly, we observed that delayed pharmacological inhibition of spinal sTNF $\alpha$ signaling dramatically attenuated SCIinduced immune suppression (Figs. 4,6). T3Tx-Saline animals became noticeably sicker after $S$. pneumoniae infection than uninjured or T3Tx-XPro1595 animals_-some even succumbed to the infection. These data corroborate the concept that high-level SCI results in a dysregulated sympathetic system that leads to immunodeficiency. Notably, while all T3Tx-XPro1595 animals got sick, they were more capable of clearing the bacteria from their lungs and all recovered substantially better than T3TxSaline animals. The improved immunity seen in the XPro1595 animals directly correlated with the prevention of SCI-induced splenic atrophy. Moreover, the leukocyte profile in uninjured and T3Tx-Saline and -XPro1595 animals 8 weeks post-SCI (i.e., time of $S$. pneumoniae application) accurately predicted immunity against infection. XPro1595 animals had more normal levels (i.e., similar to those in naive, uninjured animals) of total splenocytes, B cells, and T cells. Fascinatingly, XPro1595-treated ani- 
mals had higher numbers of $\mathrm{T}_{\text {Reg }}$ cells, important regulators of immune function particularly in disease contexts (Sakaguchi et al., 2010; Mohr et al., 2019), than both uninjured and T3TxSaline animals. This is somewhat surprising, as increases in $\mathrm{T}_{\mathrm{Reg}}$ cells are commonly associated with inhibiting lymphocyte function (Sabbagh et al., 2018). However, despite elevations in $\mathrm{T}_{\mathrm{Reg}}$ cells, XPro1595 animals had relatively normal levels of lymphocytes that may ultimately improve immunity against infection. This apparent discrepancy is likely due to contextual differences. Many studies investigating the regulatory roles of $\mathrm{T}_{\mathrm{Reg}}$ cells are in the context of autoimmune pathologies (Chen et al., 2005; Yang et al., 2018a; Mohr et al., 2019). In a SCI setting that typically causes an overall loss of lymphocytes, $\mathrm{T}_{\text {Reg }}$ cells may function differently to increase/protect this important population. We can address the role of increased $\mathrm{T}_{\text {Reg }}$ cell number in the improved immunity we observed by selectively deleting FoxP3 cells after SCI and assessing whether XPro1595 treatment still diminishes dysimmunity. Moreover, as those living with SCI are also prone to viral infections (Soden et al., 2000; Bracchi-Ricard et al., 2016), we can also determine whether XPro1595 improves antiviral immunity after SCI to more broadly gauge how well XPro1595 attenuates SCI-induced immunodeficiency.

Dysimmunity after SCI is strongly associated with a nonspecific loss of splenic leukocytes that is thought to be driven, in part, by aberrant levels of circulating stress hormones (Lucin et al., 2009; Zhang et al., 2013; Prüss et al., 2017). Indeed, we observed increased levels of splenic CORT after injury (Fig. 5a). However, despite elevated CORT levels in both T3Tx-XPro1595 and-Saline animals, T3Tx-XPro1595 animals had normal splenocyte numbers and white pulp size (Figs. $4 c, 5 c, i, l$ ). One possibility may be that increased noradrenergic signaling after SCI, suggested by increased $\mathrm{TH}^{+}$axon density in splenic white pulp and elevated levels of NE in the spleen (Fig. 5), may play a more direct role in leukocyte toxicity. The sympathetic nervous system is critically important for regulating immune function, but not much is known as to how these mechanisms may change after injury to the CNS. Perhaps with SCI, paroxysmal spikes in NE may have toxic effects on leukocytes that then contribute, independently or in combination with circulating stress hormones, to exacerbate immunosuppression (Zhang et al., 2013). The observed increase in $\mathrm{TH}^{+}$axons after injury also indicate that plasticity of adrenergic, sympathetic postganglionic neurons also play a role in heightened sympathetic reflexes after injury. Future studies will investigate whether this is an indirect effect of sTNF $\alpha$-mediated changes to SSR circuitry.

The mitigated $\mathrm{AD}$ and immune dysfunction observed in the T3Tx-XPro1595 correlated well with histological evidence that XPro1595 diminished plasticity implicated in increasing excitability of the SSR circuit. Normally, $\mathrm{CGRP}^{+}$nociceptors predominantly synapse on neurons in superficial dorsal horn laminae that then project up the spinothalamic tract to relay nociceptive information to the brain (Bokiniec et al., 2018). We know from current and previous experiments that sTNF $\alpha$ drives plasticity of nociceptors (Wheeler et al., 2014; Mironets et al., 2018). Indeed, we saw more $\mathrm{CGRP}^{+}$afferent fibers near the central canal caudal to the SCI in lumbar cord (Fig. 1). Moreover, there is a direct correlation between CRD-induced hypertension and CGRP ${ }^{+}$immunoreactivity around the central canal $\left(F_{(1,5)}=\right.$ $\left.7.946, p=0.0372, R^{2}=0.6138\right)$. Interneurons around the central canal in lumbosacral spinal cord relay sensory information rostrally, including to the SPNs (Hofstetter et al., 2005; Hou et al., 2008). Inhibiting sTNF $\alpha$ signaling decreased the number of neurons in this region that were recruited into the SSR circuit and that responded to $\mathrm{CRD}$, an established trigger of the SSR circuit (Fig. $2 a-i$ ). This suggests that injury-induced sTNF $\alpha$ causes plasticity of CGRP ${ }^{+}$afferents onto nearby interneurons, resulting in the recruitment of these rostrally projecting, lumbar interneurons into the circuit. Additionally, injury results in more interneurons in thoracic cord near SPNs being activated by a below-level sensory stimuli to drive SPN activity and sympathetic output (Krassioukov et al., 2002; Ueno et al., 2016) (Fig. 2j-t). We found that T3Tx-XPro1595 treated animals had fewer sympathetically associated interneurons activated by CRD in close proximity to SPNs. These data demonstrate that injury-induced sTNF $\alpha$ mediates the recruitment of interneurons into the SSR circuit as well as their increased excitability.

sTNF $\alpha$ may promote neuron recruitment into the circuit by enhancing synaptic strength onto these neurons. Interestingly, sTNF $\alpha$ signaling has been associated with increasing trafficking of calcium-permeable glutamatergic receptors and decreasing levels of inhibitory GABAergic receptors at the cell membrane (Beattie et al., 2002; Stellwagen et al., 2005; Stück et al., 2012; Patel et al., 2017). Future experiments will examine the direct influence of sTNF $\alpha$ on phenotypically distinct neurons within the SSR circuit in more depth.

Another possibility is that plasticity of SSR circuits is an indirect consequence of glial mechanisms that alter synaptic connectivity (Korn et al., 2005; Domercq et al., 2006; Tilleux and Hermans, 2008; Gruber-Schoffnegger et al., 2013; Liu et al., 2017). Glia have been implicated in TNFR1-mediated effects on synaptic activity (Beattie et al., 2002; Stellwagen et al., 2005; Stück et al., 2012; Patel et al., 2017). Further experiments will dissect out the role of reactive microglia or astrocytes in SSR circuit plasticity after injury.

Unlike current FDA approved TNF-inhibitors, XPro1595 specifically targets the soluble form of TNF $\alpha$, leaving transmembrane TNF $\alpha$ signaling - which is thought to mediate repair and immune modulation (Madsen et al., 2016; Atretkhany et al., 2018; Pegoretti et al., 2018; Yang et al., 2018b) - intact. In fact, XPro1595 is already currently in clinical trials as a treatment for Alzheimer's Disease (ClinicalTrials.gov, 2019), possibly shortening the time for XPro1595 to be used as a treatment for SCI. Additionally, as other medications, e.g., baclofen and morphine, are delivered intrathecally in humans (Kofler et al., 2009; Narain et al., 2015), intrathecal delivery of XPro1595, as we did here, is feasible. We intrathecally delivered XPro1595 to best contain its actions within the CNS, as systemic use of TNF-inhibitors is commonly associated with increased infection (Raychaudhuri et al., 2009). While it is possible that some XPro1595 may have escaped the CNS into the periphery via a permeabilized blood-spinal cord barrier (Noble and Wrathall, 1989; Popovich et al., 1996) or lymphatic drainage (Aspelund et al., 2015; Louveau et al., 2015), it is unlikely that this occurred to a significant extent given that we saw improved immunity in XPro1595-treated animals.

One limitation of this study is that we do not yet fully know the effective time window for XPro1595. While we know that delaying initiation of XPro1595 treatment for $3 \mathrm{~d}$ is still effective, would XPro1595 need to be administered indefinitely or can treatment be discontinued while benefits persist? Additional studies are needed to determine optimal timing and dosing.

This study demonstrates that XPro1595 therapeutically diminishes SSR circuit plasticity to attenuate sympathetic hyperreflexia and mitigate secondary consequences of SCI-induced sympathetic dysregulation, such as immunodeficiency. To our knowledge, this would be the first prophylactic therapy that broadly improves sympathetic regulation after SCI, despite being 
delivered centrally days after injury, to attenuate life-threatening effector organ dysfunction.

\section{References}

Alan N, Ramer LM, Inskip JA, Golbidi S, Ramer MS, Laher I, Krassioukov AV (2010) Recurrent autonomic dysreflexia exacerbates vascular dysfunction after spinal cord injury. Spine J 10:1108-1117.

Aspelund A, Antila S, Proulx ST, Karlsen TV, Karaman S, Detmar M, Wiig H, Alitalo K (2015) A dural lymphatic vascular system that drains brain interstitial fluid and macromolecules. J Exp Med 212:991-999.

Atretkhany KN, Mufazalov IA, Dunst J, Kuchmiy A, Gogoleva VS, Andruszewski D, Drutskaya MS, Faustman DL, Schwabenland M, Prinz M, Kruglov AA, Waisman A, Nedospasov SA (2018) Intrinsic TNFR2 signaling in T regulatory cells provides protection in CNS autoimmunity. Proc Natl Acad Sci U S A 115:13051-13056.

Beattie EC, Stellwagen D, Morishita W, Bresnahan JC, Ha BK, Von Zastrow M, Beattie MS, Malenka RC (2002) Control of synaptic strength by glial TNFalpha. Science 295:2282-2285.

Bethea JR, Nagashima H, Acosta MC, Briceno C, Gomez F, Marcillo AE, Loor K, Green J, Dietrich WD (1999) Systemically administered interleukin-10 reduces tumor necrosis factor-alpha production and significantly improves functional recovery following traumatic spinal cord injury in rats. J Neurotrauma 16:851-863.

Bokiniec P, Zampieri N, Lewin GR, Poulet JF (2018) The neural circuits of thermal perception. Curr Opin Neurobiol 52:98-106.

Bracchi-Ricard V, Lambertsen KL, Ricard J, Nathanson L, Karmally S, Johnstone J, Ellman DG, Frydel B, McTigue DM, Bethea JR (2013) Inhibition of astroglial NF-kappaB enhances oligodendrogenesis following spinal cord injury. J Neuroinflammation 10:92.

Bracchi-Ricard V, Zha J, Smith A, Lopez-Rodriguez DM, Bethea JR, AndreanskyS (2016) Chronic spinal cord injury attenuates influenza virusspecific antiviral immunity. J Neuroinflammation 13:125.

Brandt CT, Holm D, Liptrot M, Ostergaard C, Lundgren JD, Frimodt-Møller N, Skovsted IC, Rowland IJ (2008) Impact of bacteremia on the pathogenesis of experimental pneumococcal meningitis. J Infect Dis 197: 235-244.

Brommer B, Engel O, Kopp MA, Watzlawick R, Muller S, Prüss H, Chen Y, DeVivo MJ, Finkenstaedt FW, Dirnagl U, Liebscher T, Meisel A, Schwab JM (2016) Spinal cord injury-induced immune deficiency syndrome enhances infection susceptibility dependent on lesion level. Brain 139: 692-707.

Cameron AA, Smith GM, Randall DC, Brown DR, Rabchevsky AG (2006) Genetic manipulation of intraspinal plasticity after spinal cord injury alters the severity of autonomic dysreflexia. J Neurosci 26:2923-2932.

Cerri C, Caleo M, Bozzi Y (2017) Chemokines as new inflammatory players in the pathogenesis of epilepsy. Epilepsy Res 136:77-83.

Chen Z, Herman AE, Matos M, Mathis D, Benoist C (2005) Where $\mathrm{CD} 4+\mathrm{CD} 25+\mathrm{T}$ reg cells impinge on autoimmune diabetes. J Exp Med 202:1387-1397.

Clarke HA, Dekaban GA, Weaver LC (1998) Identification of lamina V and VII interneurons presynaptic to adrenal sympathetic preganglionic neurons in rats using a recombinant herpes simplex virus type 1 . Neuroscience 85:863-872.

ClinicalTrials.gov (2019) A biomarker-directed study of XPro1595 in patients with mild to moderate Alzheimer's. Available from: https:// clinicaltrials.gov/ct2/show/NCT03943264?term $=$ NCT03943264\&draw $=$ $2 \&$ rank $=1$.

Curt A, Nitsche B, Rodic B, Schurch B, Dietz V (1997) Assessment of autonomic dysreflexia in patients with spinal cord injury. J Neurol Neurosurg Psychiatry 62:473-477.

Derbenev AV, Duale H, Rabchevsky AG, Smith BN (2010) Electrophysiological characteristics of identified kidney-related neurons in adult rat spinal cord slices. Neurosci Lett 474:168-172.

Domercq M, Brambilla L, Pilati E, Marchaland J, Volterra A, Bezzi P (2006) P2Y1 receptor-evoked glutamate exocytosis from astrocytes: control by tumor necrosis factor-alpha and prostaglandins. J Biol Chem 281:3068430696.

Donnelly DJ, Popovich PG (2008) Inflammation and its role in neuroprotection, axonal regeneration and functional recovery after spinal cord injury. Exp Neurol 209:378-388.

Duale H, Hou S, Derbenev AV, Smith BN, Rabchevsky AG (2009) Spinal cord injury reduces the efficacy of pseudorabies virus labeling of sympathetic preganglionic neurons. J Neuropathol Exp Neurol 68:168-178.

Failli V, Kopp MA, Gericke C, Martus P, Klingbeil S, Brommer B, Laginha I, Chen Y, DeVivo MJ, Dirnagl U, Schwab JM (2012) Functional neurological recovery after spinal cord injury is impaired in patients with infections. Brain 135:3238-3250.

Feldman C, Munro NC, Jeffery PK, Mitchell TJ, Andrew PW, Boulnois GJ, Guerreiro D, Rohde JA, Todd HC, Cole PJ (1991) Pneumolysin induces the salient histologic features of pneumococcal infection in the rat lung in vivo. Am J Respir Cell Mol Biol 5:416-423.

Garshick E, Kelley A, Cohen SA, Garrison A, Tun CG, Gagnon D, Brown R (2005) A prospective assessment of mortality in chronic spinal cord injury. Spinal Cord 43:408-416.

Gruber-Schoffnegger D, Drdla-Schutting R, Hönigsperger C, Wunderbaldinger G, Gassner M, Sandkühler J (2013) Induction of thermal hyperalgesia and synaptic long-term potentiation in the spinal cord lamina I by TNF-alpha and IL-1beta is mediated by glial cells. J Neurosci 33:6540-6551.

Hofstetter CP, Card JP, Olson L (2005) A spinal cord pathway connecting primary afferents to the segmental sympathetic outflow system. Exp Neurol 194:128-138.

Hou S, Duale H, Cameron AA, Abshire SM, Lyttle TS, Rabchevsky AG (2008) Plasticity of lumbosacral propriospinal neurons is associated with the development of autonomic dysreflexia after thoracic spinal cord transection. J Comp Neurol 509:382-399.

Hou S, Duale H, Rabchevsky AG (2009) Intraspinal sprouting of unmyelinated pelvic afferents after complete spinal cord injury is correlated with autonomic dysreflexia induced by visceral pain. Neuroscience 159:369379.

Hou S, Tom VJ, Graham L, Lu P, Blesch A (2013) Partial restoration of cardiovascular function by embryonic neural stem cell grafts after complete spinal cord transection. J Neurosci 33:17138-17149.

Joshi S, Levatte MA, Dekaban GA, Weaver LC (1995) Identification of spinal interneurons antecedent to adrenal sympathetic preganglionic neurons using trans-synaptic transport of herpes simplex virus type 1. Neuroscience 65:893-903.

Kigerl KA, Hall JC, Wang L, Mo X, Yu Z, Popovich PG (2016) Gut dysbiosis impairs recovery after spinal cord injury. J Exp Med 213:2603-2620.

Kofler M, Poustka K, Saltuari L (2009) Intrathecal baclofen for autonomic instability due to spinal cord injury. Auton Neurosci 146:106-110.

Korn T, Magnus T, Jung S (2005) Autoantigen specific T cells inhibit glutamate uptake in astrocytes by decreasing expression of astrocytic glutamate transporter GLAST: a mechanism mediated by tumor necrosis factoralpha. FASEB J 19:1878-1880.

Krassioukov A, Claydon VE (2006) The clinical problems in cardiovascular control following spinal cord injury: an overview. Prog Brain Res 152: 223-229.

Krassioukov AV, Weaver LC (1995) Episodic hypertension due to autonomic dysreflexia in acute and chronic spinal cord-injured rats. Am J Physiol 268:H2077-H2083.

Krassioukov AV, Johns DG, Schramm LP (2002) Sensitivity of sympathetically correlated spinal interneurons, renal sympathetic nerve activity, and arterial pressure to somatic and visceral stimuli after chronic spinal injury. J Neurotrauma 19:1521-1529.

Krenz NR, Weaver LC (1998) Sprouting of primary afferent fibers after spinal cord transection in the rat. Neuroscience 85:443-458.

Krenz NR, Meakin SO, Krassioukov AV, Weaver LC (1999) Neutralizing intraspinal nerve growth factor blocks autonomic dysreflexia caused by spinal cord injury. J Neurosci 19:7405-7414.

Kroner A, Greenhalgh AD, Zarruk JG, Passos Dos Santos R, Gaestel M, David S (2014) TNF and increased intracellular iron alter macrophage polarization to a detrimental M1 phenotype in the injured spinal cord. Neuron 83:1098-1116.

Landrum LM, Jones SL, Blair RW (2002) The expression of fos-labeled spinal neurons in response to colorectal distension is enhanced after chronic spinal cord transection in the rat. Neuroscience 110:569-578.

Lee YL, Shih K, Bao P, Ghirnikar RS, Eng LF (2000) Cytokine chemokine expression in contused rat spinal cord. Neurochem Int 36:417-425.

Liu Y, Zhou LJ, Wang J, Li D, Ren WJ, Peng J, Wei X, Xu T, Xin WJ, Pang RP, Li YY, Qin ZH, Murugan M, Mattson MP, Wu LJ, Liu XG (2017) TNFalpha differentially regulates synaptic plasticity in the hippocampus and 
spinal cord by microglia-dependent mechanisms after peripheral nerve injury. J Neurosci 37:871-881.

Louveau A, Smirnov I, Keyes TJ, Eccles JD, Rouhani SJ, Peske JD, Derecki NC, Castle D, Mandell JW, Lee KS, Harris TH, Kipnis J (2015) Structural and functional features of central nervous system lymphatic vessels. Nature 523:337-341.

Lucin KM, Sanders VM, Popovich PG (2009) Stress hormones collaborate to induce lymphocyte apoptosis after high level spinal cord injury. J Neurochem 110:1409-1421.

MacDonald KD, Chang HY, Mitzner W (2009) An improved simple method of mouse lung intubation. J Appl Physiol 106:984-987.

Madsen PM, Motti D, Karmally S, Szymkowski DE, Lambertsen KL, Bethea JR, Brambilla R (2016) Oligodendroglial TNFR2 mediates membrane TNF-dependent repair in experimental autoimmune encephalomyelitis by promoting oligodendrocyte differentiation and remyelination. J Neurosci 36:5128-5143.

Marsh DR, Wong ST, Meakin SO, MacDonald JI, Hamilton EF, Weaver LC (2002) Neutralizing intraspinal nerve growth factor with a trkA-IgG fusion protein blocks the development of autonomic dysreflexia in a clipcompression model of spinal cord injury. J Neurotrauma 19:1531-1541.

Matsushita M (1998) Ascending propriospinal afferents to area X (substantia grisea centralis) of the spinal cord in the rat. Exp Brain Res 119:356-366.

Mayorov DN, Adams MA, Krassioukov AV (2001) Telemetric blood pressure monitoring in conscious rats before and after compression injury of spinal cord. J Neurotrauma 18:727-736.

Mironets E, Osei-Owusu P, Bracchi-Ricard V, Fischer R, Owens EA, Ricard J, Wu D, Saltos T, Collyer E, Hou S, Bethea JR, Tom VJ (2018) Soluble TNFalpha signaling within the spinal cord contributes to the development of autonomic dysreflexia and ensuing vascular and immune dysfunction after spinal cord injury. J Neurosci 38:4146-4162.

Mohr A, Atif M, Balderas R, Gorochov G, Miyara M (2019) The role of FOXP3 $(+)$ regulatory $\mathrm{T}$ cells in human autoimmune and inflammatory diseases. Clin Exp Immunol 197:24-35.

Narain S, Al-Khoury L, Chang E (2015) Resolution of chronic migraine headaches with intrathecal ziconotide: a case report. J Pain Res 8: 603-606.

Noble LJ, Wrathall JR (1989) Distribution and time course of protein extravasation in the rat spinal cord after contusive injury. Brain Res 482:57-66.

Partida E, Mironets E, Hou S, Tom VJ (2016) Cardiovascular dysfunction following spinal cord injury. Neural Regen Res 11:189-194.

Patel DC, Wallis G, Dahle EJ, McElroy PB, Thomson KE, Tesi RJ, Szymkowski DE, West PJ, Smeal RM, Patel M, Fujinami RS, White HS, Wilcox KS (2017) Hippocampal TNFalpha signaling contributes to seizure generation in an infection-induced mouse model of limbic epilepsy. eNeuro 4:ENEURO.0105-17.2017.

Pegoretti V, Baron W, Laman JD, Eisel ULM (2018) Selective modulation of TNF-TNFRs signaling: insights for multiple sclerosis treatment. Front Immunol 9:925.

Popovich PG, Horner PJ, Mullin BB, Stokes BT (1996) A quantitative spatial analysis of the blood-spinal cord barrier. I. permeability changes after experimental spinal contusion injury. Exp Neurol 142:258-275.

Prüss H, Tedeschi A, Thiriot A, Lynch L, Loughhead SM, Stutte S, Mazo IB, Kopp MA, Brommer B, Blex C, Geurtz LC, Liebscher T, Niedeggen A, Dirnagl U, Bradke F, Volz MS, DeVivo MJ, Chen Y, von Andrian UH, Schwab JM (2017) Spinal cord injury-induced immunodeficiency is mediated by a sympathetic-neuroendocrine adrenal reflex. Nat Neurosci 20:1549-1559.

Rabchevsky AG, Patel SP, Duale H, Lyttle TS, O’Dell CR, Kitzman PH (2011) Gabapentin for spasticity and autonomic dysreflexia after severe spinal cord injury. Spinal Cord 49:99-105.

Rabchevsky AG, Patel SP, Lyttle TS, Eldahan KC, O’Dell CR, Zhang Y, Popovich PG, Kitzman PH, Donohue KD (2012) Effects of gabapentin on muscle spasticity and both induced as well as spontaneous autonomic dysreflexia after complete spinal cord injury. Front Physiol 3:329.

Raychaudhuri SP, Nguyen CT, Raychaudhuri SK, Gershwin ME (2009) In- cidence and nature of infectious disease in patients treated with anti-TNF agents. Autoimmun Rev 9:67-81.

Sabbagh P, Karkhah A, Nouri HR, Javanian M, Ebrahimpour S (2018) The significance role of regulatory $\mathrm{T}$ cells in the persistence of infections by intracellular bacteria. Infect Genet Evol 62:270-274.

Sakaguchi S, Miyara M, Costantino CM, Hafler DA (2010) FOXP3 + regulatory $\mathrm{T}$ cells in the human immune system. Nat Rev Immunol 10: $490-500$.

Sauerbeck AD, Laws JL, Bandaru VV, Popovich PG, Haughey NJ, McTigue DM (2015) Spinal cord injury causes chronic liver pathology in rats. J Neurotrauma 32:159-169.

Soden RJ, Walsh J, Middleton JW, Craven ML, Rutkowski SB, Yeo JD (2000) Causes of death after spinal cord injury. Spinal Cord 38:604-610.

Steed PM, et al. (2003) Inactivation of TNF signaling by rationally designed dominant-negative TNF variants. Science 301:1895-1898.

Stellwagen D, Beattie EC, Seo JY, Malenka RC (2005) Differential regulation of AMPA receptor and GABA receptor trafficking by tumor necrosis factor-alpha. J Neurosci 25:3219-3228.

Stück ED, Christensen RN, Huie JR, Tovar CA, Miller BA, Nout YS, Bresnahan JC, Beattie MS, Ferguson AR (2012) Tumor necrosis factor alpha mediates GABA (A) receptor trafficking to the plasma membrane of spinal cord neurons in vivo. Neural Plast 2012:261345.

Tang X, Neckel ND, Schramm LP (2004) Spinal interneurons infected by renal injection of pseudorabies virus in the rat. Brain Res 1004:1-7.

Tilleux S, Hermans E (2008) Down-regulation of astrocytic GLAST by microglia-related inflammation is abrogated in dibutyryl cAMPdifferentiated cultures. J Neurochem 105:2224-2236.

Ueno M, Ueno-Nakamura Y, Niehaus J, Popovich PG, Yoshida Y (2016) Silencing spinal interneurons inhibits immune suppressive autonomic reflexes caused by spinal cord injury. Nat Neurosci 19:784-787.

Wang CX, Nuttin B, Heremans H, Dom R, Gybels J (1996) Production of tumor necrosis factor in spinal cord following traumatic injury in rats. J Neuroimmunol 69:151-156.

Weaver LC, Verghese P, Bruce JC, Fehlings MG, Krenz NR, Marsh DR (2001) Autonomic dysreflexia and primary afferent sprouting after clipcompression injury of the rat spinal cord. J Neurotrauma 18:1107-1119.

Weaver LC, Marsh DR, Gris D, Meakin SO, Dekaban GA (2002) Central mechanisms for autonomic dysreflexia after spinal cord injury. Prog Brain Res 137:83-95.

West CR, Popok D, Crawford MA, Krassioukov AV (2015) Characterizing the temporal development of cardiovascular dysfunction in response to spinal cord injury. J Neurotrauma 32:922-930.

West CR, Crawford MA, Laher I, Ramer MS, Krassioukov AV (2016) Passive hind-limb cycling reduces the severity of autonomic dysreflexia after experimental spinal cord injury. Neurorehabil Neural Repair 30:317-327.

Wheeler MA, Heffner DL, Kim S, Espy SM, Spano AJ, Cleland CL, Deppmann CD (2014) TNF-alpha/TNFR1 signaling is required for the development and function of primary nociceptors. Neuron 82:587-602.

Wu D, Klaw MC, Connors T, Kholodilov N, Burke RE, Tom VJ (2015) Expressing constitutively active rheb in adult neurons after a complete spinal cord injury enhances axonal regeneration beyond a chondroitinase-treated glial scar. J Neurosci 35:11068-11080.

Xu C, Klaw MC, Lemay MA, Baas PW, Tom VJ (2015) Pharmacologically inhibiting kinesin-5 activity with monastrol promotes axonal regeneration following spinal cord injury. Exp Neurol 263:172-176.

Yang M, Klocke K, Hernandez CM, Xu B, Gjertsson I, Wing K, Holmdahl R (2018a) Regulatory $\mathrm{T}$ cells control epitope spreading in autoimmune arthritis independent of cytotoxic T-lymphocyte antigen-4. Immunology 155:446-457.

Yang S, Wang J, Brand DD, Zheng SG (2018b) Role of TNF-TNF receptor 2 signal in regulatory $\mathrm{T}$ cells and its therapeutic implications. Front Immunol 9:784.

Zhang Y, Guan Z, Reader B, Shawler T, Mandrekar-Colucci S, Huang K, Weil Z, Bratasz A, Wells J, Powell ND, Sheridan JF, Whitacre CC, Rabchevsky AG, Nash MS, Popovich PG (2013) Autonomic dysreflexia causes chronic immune suppression after spinal cord injury. J Neurosci 33: $12970-12981$. 